\title{
Demonstration of deployment repeatability of key subsystems of a furled starshade architecture
}

\author{
Manan Arya $\odot$, ,** Flora Mechentel, ${ }^{\mathrm{a}}$ David Webb, ${ }^{\mathrm{a}}$ John Steeves $\odot,{ }^{\mathrm{a}}$ \\ Doug Lisman, ${ }^{a}$ Stuart Shaklan $\odot,{ }^{a}$ Samuel Case Bradford, ${ }^{a}$ Eric Kelso, \\ Kenzo Neff, ${ }^{b}$ Amanda Swain, ${ }^{b}$ Andrei Iskra, ${ }^{b}$ Neal Beidleman,

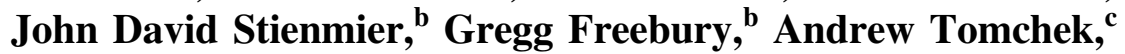 \\ Tayler Thomas, ${ }^{\mathrm{c}}$ Craig Hazelton, ${ }^{\mathrm{c}}$ Kassi Butler, ${ }^{\mathrm{c}}$ Kamron Medina, \\ Mike Pulford, ${ }^{c}$ Larry Adams, ${ }^{c}$ David Hepper, ${ }^{c}$ and Dana Turse ${ }^{c}$ \\ ${ }^{a}$ Jet Propulsion Laboratory, California Institute of Technology, Pasadena, California, \\ United States \\ ${ }^{\mathrm{b}}$ Tendeg, Louisville, Colorado, United States \\ ${ }^{c}$ Roccor, Longmont, Colorado, United States
}

\begin{abstract}
Starshade concepts must be stowed within rocket fairings for launch and then deployed in space. The in-plane deployment accuracy must be on the order of hundreds of micrometers for sufficient starlight suppression to enable the detection and study of Earth-like exoplanets around nearby Sun-like stars. We describe tests conducted to demonstrate deployment repeatability of two key structural subsystems of the "furled" starshade architecture- the petal and the inner disk. Together, the petals and the inner disk create the in-plane shape of a starshade. Test articles to represent the petal and inner disk subsystems were constructed at relevant scales for a 26-m-diameter starshade. These test articles were subjected to stowageand-deployment cycles and their shapes were measured. The measured performance-tens of parts per million of petal strain after deployment, and hundreds of micrometers of inner disk deployment accuracy — was found to be within required allocations. (C) The Authors. Published by SPIE under a Creative Commons Attribution 4.0 Unported License. Distribution or reproduction of this work in whole or in part requires full attribution of the original publication, including its DOI. [DOI: 10 .1117/1.JATIS.7.2.021202]
\end{abstract}

Keywords: starshades; high-contrast imaging; deployable structures; deployment accuracy.

Paper 20106SS received Jul. 29, 2020; accepted for publication Nov. 6, 2020; published online Jan. 6, 2021.

\section{Introduction}

The direct imaging of exoplanets is key to their study; however, such direct imaging is challenging given the small angular separation between exoplanets and their host stars and the large difference in the magnitude of light from these objects. Starlight suppression-the reduction of the relative brightness of the host star-is critical.

Starshades are a promising mechanism for starlight suppression. ${ }^{1}$ A starshade is a screen external to a telescope that blocks starlight before it enters the telescope. The shape of a starshade is designed to reduce starlight diffracted into the telescope. Starshades could achieve the suppression necessary for the direct imaging of Earth-like exoplanets. ${ }^{2}$

The key challenges in the mechanical engineering of starshades stem from two requirements: (1) the starshade diameter must be $\sim 30 \mathrm{~m}$ to shade $\sim 2$-m-diameter space-based telescope apertures (whereas the largest rocket fairings are only 5.4-m in diameter); and (2) to achieve sufficient starlight suppression to detect Earth-like exoplanets, the deployed in-plane shape of a starshade must be accurate to within hundreds of microns. As such, a starshade must be folded to fit inside a rocket fairing for launch, and then unfolded to an accurate shape in space.

When the shape deviates from the design, starlight leaks through and appears in the image plane, potentially veiling the planet. The ratio of the leaked light to the peak of the starlight is

*Address all correspondence to Manan Arya, manan.arya@jpl.nasa.gov 
termed the "instrument contrast." The goal of the starshade design is to minimize the instrument contrast to values below $1 \times 10^{-10}$.

NASA's Exoplanet Exploration Program has an activity called S5 to mature starshade technology to technology readiness level 5 (TRL5). ${ }^{2} \mathrm{~S} 5$ is structured around a number of technology milestones. This paper describes tests conducted to satisfy milestones related to the mechanical deployment accuracy of a "furled" starshade architecture. These tests demonstrate the deployment performance required to detect and study Earth-like exoplanets in the habitable zones of nearby Sun-like stars.

Portions of this paper are repeated verbatim from a conference proceedings $\operatorname{paper}^{3}$ and technical reports written for the S5 activity.,

This paper is laid out as follows: Sec. 1 introduces the starshade mechanical design architecture and lists the objectives of this work. Section 2 describes the test articles. Section 3 describes the test apparatus, and Sec. 4 describes the test procedures. Section 5 defines the data processing and analysis methods used to reduce the measured data. Section 6 shows the test results, and Sec. 7 discusses the test results. Finally, Sec. 8 provides concluding remarks.

\subsection{Starshade Mechanical Design}

Following the S5 Technology Development Plan, ${ }^{2}$ this paper uses the Starshade Rendezvous Mission (SRM) concept as a reference design. As shown in Fig. 1, the SRM starshade is 26-m in diameter, with 248 -m-long petals attached to a 10 -m-diameter inner disk. ${ }^{6}$ The SRM concept is to launch a starshade to rendezvous with the Nancy Grace Roman Space Telescope in a halo orbit around the Sun-Earth L2. This paper is also relevant to the Habitable Exoplanet Observatory (HabEx) concept, ${ }^{7}$ for which the starshade uses the same deployment architecture but is double in size-52-m in diameter with 24 16-m-long petals and a 20-m-diameter inner disk.

The stowed inner disk has a barrel-like form around which the petals are wrapped. Figure 1 shows the two steps of starshade deployment: first, the petals unfurl, and second, the inner disk unfolds. During the first step of petal unfurling, the stowed inner disk remains latched in position. During the second step of inner disk unfolding, the petals are fully deployed and have

Step 1: petal unfurling

(a)

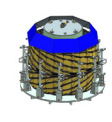

(b)

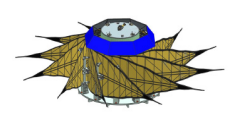

(c)

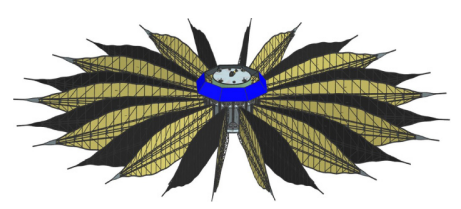

(d)

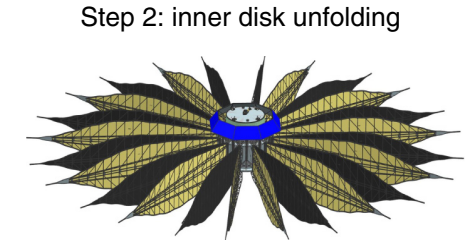

(e)
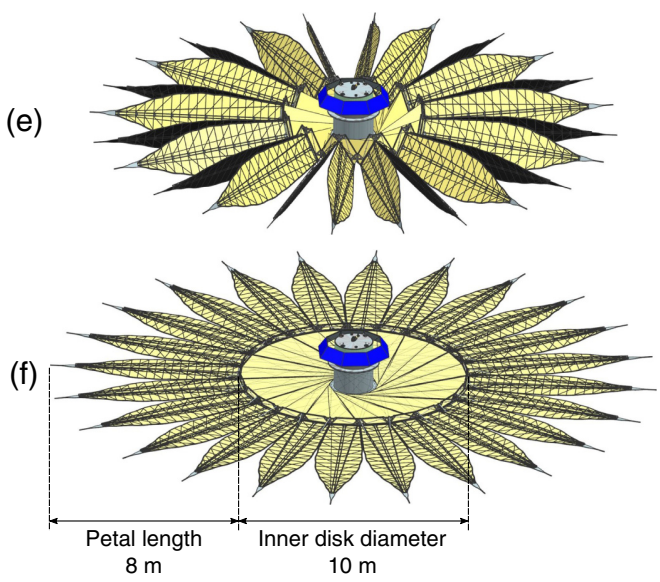

Fig. 1 Starshade deployment concept for the SRM design. Step 1 is petal unfurling, which is controlled by a carousel of rollers. At the end of step 1, shown in (c), the carousel is jettisoned. Step 2 is inner disk unfolding, in which the petals are deployed to their operational position. (a) Stowed, (b) petals partially unfurled, (c) petals unfurled and carousel jettisoned, (d) start of inner disk deployment, (e) inner disk partially deployed, and (f) starshade deployed. 
independent structural stiffness. The petals and the inner disk are exercised in sequence with a clean interface between them, and thus these two deployment steps can be studied independently. This starshade mechanical architecture has been described previously; ${ }^{8-12}$ here it will be summarized in brief.

Each petal has a planar truss of thermally stable carbon-fiber-composite members that maintains the petal width profile. Having accurate and stable petal width profiles is critical to minimizing instrument contrast degradation. The petals are furled such that the resulting bending strains have negligible effect on the petal width. Two "pop-up" ribs on each petal provide out-ofplane bending stiffness when deployed. For furling, these ribs fold to lie flat and furl with the planar petal truss.

Petal unfurling is driven by stored strain energy. A "carousel" of rollers constrains the petals during unfurling to provide controlled and quasi-static deployment. A motor rotates this carousel with respect to petals and the petals gradually unfurl. Once unfurled, the two spring-loaded "popup" ribs on each petal unfold. At the end of petal unfurling, the rollers hinge away from the petals and the roller carousel is jettisoned.

The deployed petal positions are set by the inner disk, which is a stiff preloaded structure, very much like a bicycle wheel: a number of tension spokes connect a central hub to a perimeter truss. The spoke tension is reacted by the compression of the perimeter truss and the hub. A multi-layer optical shield rests below all the spokes and provides opacity.

The perimeter truss stows in a circular $z$-fold, going from a deployed ring to a stowed barrel. The optical shield folds in an origami wrapping pattern, occupying the space between the hub and the stowed perimeter truss. A motor on the perimeter truss drives inner disk deployment. The optical shield and the spokes are passively unfolded by the perimeter truss.

This starshade mechanical architecture draws heritage from successful deployable antenna reflector technologies for spacecraft. The petal unfurling adopts the deployment approach of wrap-rib antennas, which have been deployed in space a number of times. ${ }^{13}$ Wrap-rib antennas consist of a mesh reflector supported by radial ribs. The ribs are wrapped around a central hub for stowage and deploy using stored strain energy. The inner disk structural architecture draws from the design of hoop/column reflectors, which have been extensively tested. ${ }^{14}$ Hoop/column reflectors are preloaded structures consisting of a perimeter hoop, a central column, and a number of tension spokes that connect them. Additionally, the inner disk perimeter truss draws heritage from the trusses of the AstroMesh ${ }^{\circledR}$ antenna reflectors, ${ }^{15}$ which have been deployed in space several times.

In 2012, Kasdin et al. ${ }^{16}$ demonstrated the manufacturing, assembly, and metrology of a 6-m-long, 2.3-m-wide starshade petal to $\pm 100 \mu \mathrm{m}$ in-plane accuracy. However, they did not demonstrate petal shape recovery after stowage and deployment. In 2014, Kasdin et al. ${ }^{17,18} \mathrm{dem}-$ onstrated the deployment of a 12-m-diameter inner disk with four petals. The deployment accuracy of the petals was measured to be $\pm 250 \mu \mathrm{m}$ in the radial direction and $\pm 500 \mu \mathrm{m}$ in the tangential direction. However, this test did not include the optical shield and used a perimeter truss from an AstroMesh ${ }^{\circledR}$ antenna, which is not flight-like for a starshade. In 2016, Hirsch et al. ${ }^{19}$ constructed a starshade-compatible perimeter truss; however, this prototype was unable to demonstrate sufficient deployment accuracy.

This paper builds on the previous work and fills important gaps by demonstrating the ability of a starshade petal to recover its shape after stowage and deployment cycles and the deployment accuracy of an inner disk with a flight-like perimeter truss and an optical shield.

\subsection{Objectives and Approach}

This paper has two objectives: (1) to demonstrate that a starshade petal can recover its as-manufactured shape to specified tolerances after unfurling; and (2) to demonstrate that the starshade inner disk can deploy to position the petals to specified levels of accuracy.

To meet these objectives, an experimental approach was taken. A petal test article was constructed, subjected to a number of stowage-and-deployment cycles, and its resulting shape change was measured. Similarly, an inner disk test article was assembled, subjected to a number of deployment cycles, and its deployment accuracy was characterized. All testing was conducted in a Tendeg facility in Louisville, Colorado. 
The requirements on the petal shape recovery tolerance and the inner disk deployment accuracy are based on an error budget with the goal of detecting and characterizing exo-Earths. ${ }^{2}$ This error budget allocates degradation in instrument contrast to a number of sources, and is an upgraded version of the one presented in Refs. 6 and 20. The requirements are as follows.

- The petal test article must return to its manufactured shape such that the petal width strain bias is within $\pm 26 \mathrm{ppm}$ after a number of deployment cycles.

- The inner disk test article must deploy such that the critical components of the petal position accuracy errors - radial bias error, radial random error, tangential random error, and clocking random error-are $<35 \mu \mathrm{m}, 150 \mu \mathrm{m}, 120 \mu \mathrm{m}$, and $180 \mu \mathrm{rad}$, respectively, at the $3 \sigma$ level.

Petal width strain bias is the zeroth-order width strain of a petal over its length and predicts well the degradation in instrument contrast due to petal shape changes. The petal position accuracy errors describe the in-plane rigid-body translations and rotations of the petals. Radial bias and radial random errors capture the petal translations in the radial direction, tangential random error captures translations in the tangential direction, and clocking random captures in-plane petal rotations. (Tangential bias and clocking bias have negligible effect on instrument contrast degradation.)

\section{Test Articles}

\subsection{Petal Test Article}

Figure 2 shows the SRM petal design. It is $8-\mathrm{m}$ long, with a width of $1.3 \mathrm{~m}$ at the base, and a maximum width of $2 \mathrm{~m}$. Three hinges at the base of the petal are the interface to the inner disk perimeter truss.

The petal has a planar carbon-fiber-reinforced-polymer (CFRP) truss structure that provides an accurate and stable width profile-the distance between the two terminal edges over the length of the petal. Key members in this structure are the battens, which span the petal width; the braces, which are arranged diagonally for shear stiffness; and the petal spine, which provides longitudinal stiffness. A continuous CFRP structure on the perimeter of the petal supports the

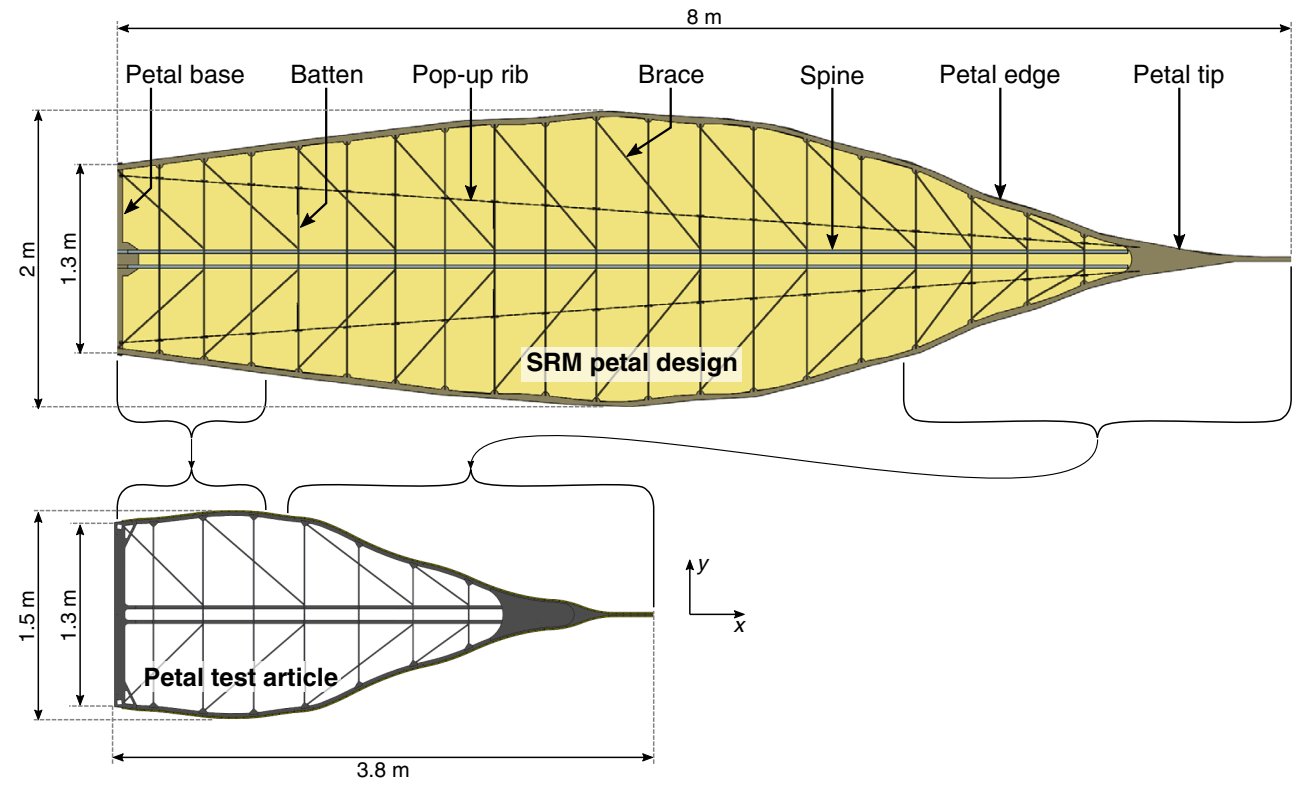

Fig. 2 Petal design for the SRM concept compared to the petal test article constructed for the present effort. Inboard and outboard sections of the SRM petal are conjoined to form the petal test article. 
terminal edge, which is a $38-\mu \mathrm{m}$-thick amorphous metal foil. The foil is photochemically etched to an accurate in-plane shape and a sharp terminal radius to minimize solar scatter (see Refs. 2123); 1-m-long segments of the etched foil are bonded to the petal CFRP structure.

Two "pop-up" ribs provide additional out-of-plane stiffness for each petal. Each rib is hinged to the petal using sliding hinges that allow relative translation along the hinge axis. This decouples the precision in-plane petal structure from the rib. For stowage, the ribs fold flat against the petal and wrap with the petal. Upon deployment, spring-loaded struts cause the ribs to pop-up.

The SRM petal design has a number of launch restraints attached to the spine. Upon furling, these restraints nestle into identical restrains on adjacent petals. For launch, compression loads are applied to each stack of petal launch restraints to lock the petals in a furled configuration.

A lightweight multi-layer opacity blanket covers the petal CFRP structure. The opacity blanket is nominally non-tensioned, and it is connected to the precision in-plane petal structure using compliant $z$-folded zones. These design features minimize the coupling between the the opacity blanket and the precision petal structure.

Figure 2 also shows the design of the petal test article, which includes the planar CFRP truss structure. The materials and the cross sections of the members of the test article are representative of the SRM design, as are the geometries of the joints between the members. The ribs, the launch restraints, and the opacity blanket are omitted in this test article. These components are designed to have minimal influence on the petal width profile: the ribs are connected to the petal structure using sliding hinges and their shallow angles to the $x$ axis reduce their projected stiffness along the width-wise $y$ axis; the launch restraints attached to the petal spines are relatively small (thus their stiffness is localized) and they have minimal influence on the petal width; and the opacity blanket is non-tensioned and attached to the petal structure using compliant connections. In a step-wise experimental approach, this work demonstrates the deployment repeatability of the critical widthpreserving petal structure; future testing will employ test articles that have all components.

The petal test article has 3.8-m length, 1.3-m width at the base, and a maximum width of $1.5 \mathrm{~m}$. Compared to the SRM design, the petal test article is $1 / 2$-scale in length and $3 / 4$-scale in maximum width. The size of the petal test article is driven by limitations of the metrology system (see Sec. 3.3.1). To maintain the design commonality to the SRM petal, the petal test article is a concatenation of the base and tip sections of the SRM petal, as shown in Fig. 2.

Flight-like materials were used to construct the petal test article. The edges, the spine, the base, and the tip consist of CFRP plates (Toray ${ }^{\circledR}$ M55J carbon fiber laminates with cyanate ester matrix). The battens and the braces consist of machine-drawn uniaxially pultruded carbon-fiber-epoxy rods. (Uniaxial pultrusion ensures uniformity of material and cross-section properties along the length and consistency across production lots.) A two-part room-temperature curing epoxy (Loctite ${ }^{\circledR}$ EA9394) was used to bond the members together. Figure 3 shows the petal test article.

The in-plane shape of the petal test article was not controlled to flight-like tolerances since flight-like manufacturing accuracy has been demonstrated in Ref. 16. The objective of this paper is to demonstrate recovery of petal shape after stow-and-deploy cycles, which does not require absolute accuracy.

The petal test article replicates key aspects of the SRM petal design: a portion at the base of the petal, a portion at the tip of the petal, and a number of intervening repeated truss-like units (each unit consisting of two battens, two braces, and lengths of the petal edges and the petal spines). In both, the SRM design and the test article, these repeated truss-like units have variations from one instance to the next, primarily in the width of the unit. However, given the commonalities between these units- the structural architecture, the materials and dimensions of the constituent members, and the applied loading — each unit is expected to have similar deployment repeatability. (Additionally, the key performance metric is the dimensionless width strain, which is independent of width.) Since the petal test article has a number of these units, it is expected to capture the physical mechanisms that may cause petal shape change from deployment-to-deployment.

\subsection{Inner Disk Subsystem Test Article}

Figure 4 shows an as-designed overview of the inner disk testbed, which consists of the inner disk test article, metrology hardware, and gravity compensation devices. 


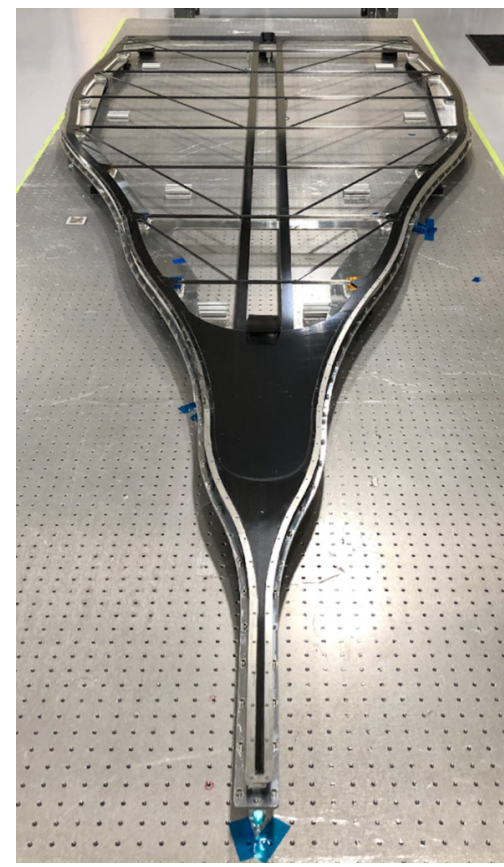

Fig. 3 The petal test article.

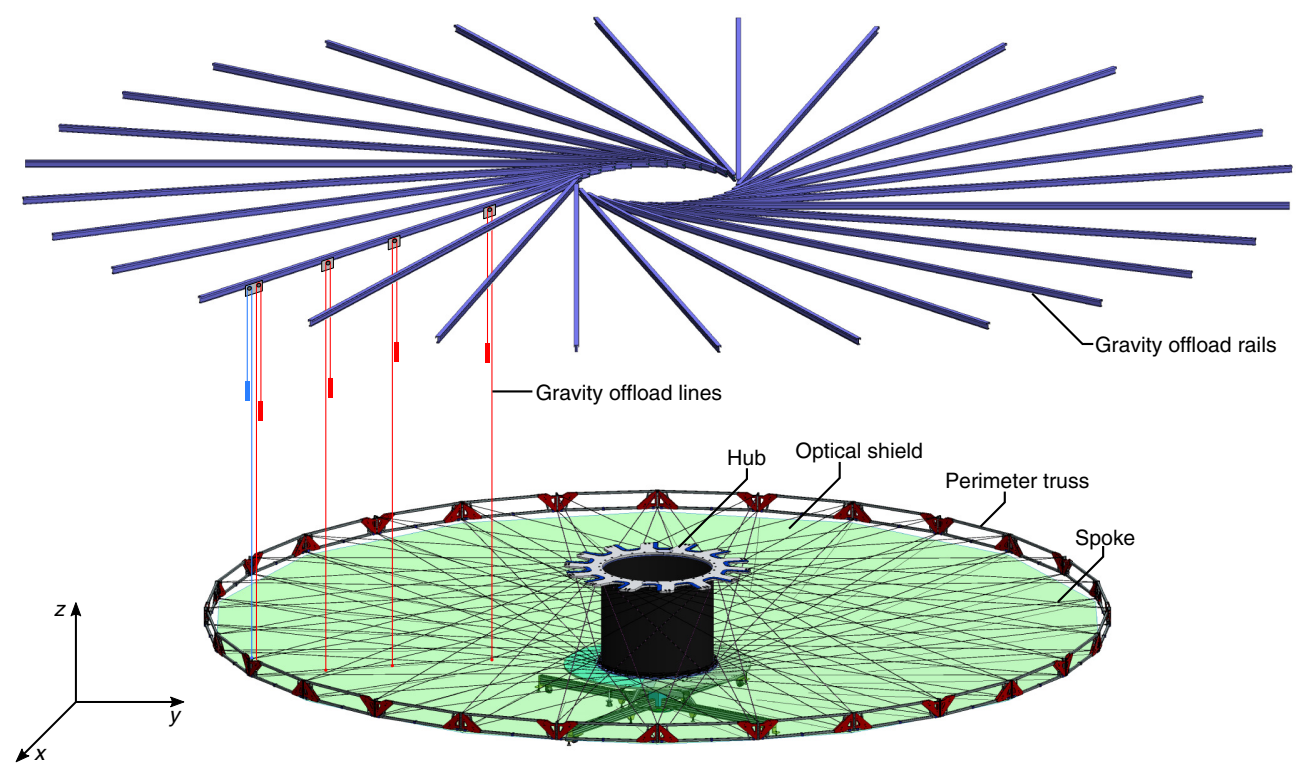

Fig. 4 Overview of the inner disk testbed. The optical shield, in green, is transparent for the sake of clarity. Only one set of gravity offload lines are shown, again, for clarity. In reality, each of the 28 gravity offload rails supports four offload lines. The inner disk test article is oriented with the telescope side facing down. The $z$ axis of the coordinate system is aligned with gravity.

The inner disk test article is full-scale for SRM. The deployed diameter of the inner disk test article is $10.6 \mathrm{~m}$-comparable to the $9.8 \mathrm{~m}$ deployed diameter of the SRM inner disk. The stowed diameter of the inner disk test article is $2.3 \mathrm{~m}$, identical to the stowed diameter of the SRM inner disk.

An important difference between the inner disk test article and the SRM design is the number of petals: the SRM design has 24 petals, but the test article has a perimeter truss designed for 28 petals. This is because the inner disk test article utilizes a 28 -sided perimeter truss that was constructed before the baseline SRM design was established. To expedite schedule, this existing 
(a)

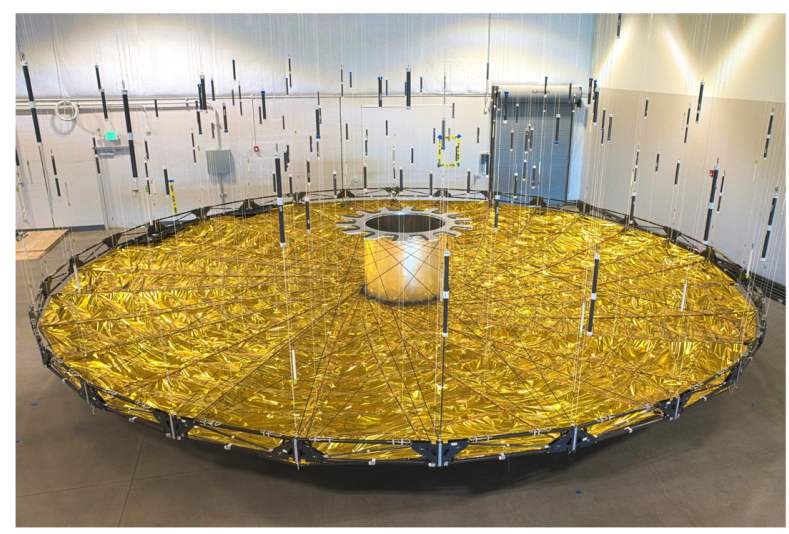

(b)

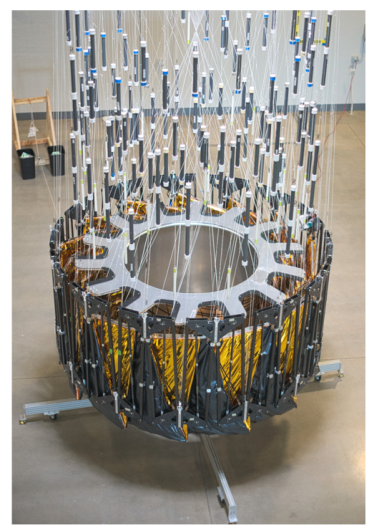

Fig. 5 The inner disk test article (a) deployed and (b) stowed. Deployed, the test article is 10.6-m in diameter. Stowed, it is 2.3- $\mathrm{m}$ in diameter. The two images are not at the same scale. A number of gravity offload counterweights are visible in both images.

perimeter truss was modified and used for this experiment. It is expected that the results from this experiment are applicable to the SRM design. On a local level (i.e., at every unit cell of the rotationally symmetric structure), the test article and the SRM inner disk are similar. At a global level, the deployment accuracy of the inner disk is not expected to vary between 24 and 28 petals.

Figure 5 shows photographs of the inner disk test article. Below are detailed the design and construction of its individual components: the perimeter truss, the spokes, the hub, and the optical shield.

\subsubsection{Perimeter truss}

The perimeter truss is a stiff structure that reacts the tensile preload of the spokes in the deployed configuration. It provides attachment interfaces for each of the 28 petals. Deployed, it is $10.6-\mathrm{m}$ in diameter.

The perimeter truss is rotationally symmetric, consisting of 28 bays. Each bay is a four-bar linkage - four rigid members arranged in a planar parallelogram, linked to each other through revolute joints. (A revolute joint is a single-degree-of-freedom joint that allows for free rotation about a fixed axis, e.g., a scissor joint.) Figure 6 shows a single truss bay, with the four-bar linkage highlighted in green. By exercising the shear mechanism of each of the 28 four-bar linkages, the perimeter truss can transition from its deployed ring-like geometry to a stowed barrel-like form, as shown in Fig. 7. The longeron and the shorteron are two of the bars of this linkage. (The term "longeron" is commonly used in structural engineering for a load-bearing member in a framework. The term "shorteron" is specific to this starshade design: a shorteron is similar to a longeron, except shorter, hence "shorteron.") They are connected to a node- a stiff triangular structure-at either end through revolute joints. The sides of the nodes form the other two bars of the four-bar linkage.

Along a diagonal of each bay is a pair of telescoping tubes. As this diagonal shortens during deployment, the inner tube slides into the outer tube. Pawls and ratchets on these tubes prevent a bay from being driven backward. A single braided steel drive cable is strung across all bays.

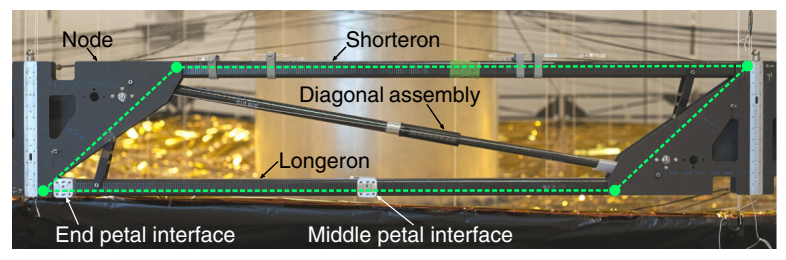

Fig. 6 A single-perimeter truss bay, showing key components of the four-bar linkage mechanism that actuates each bay. The four-bar linkage is highlighted in green. 


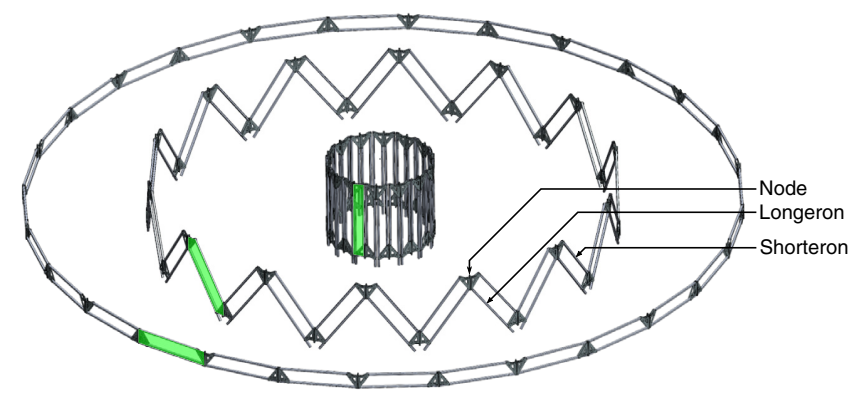

Fig. 7 Folding and deployment kinematics of the perimeter truss. A single bay is highlighted in green. Note that the diagonal assemblies are omitted in this figure.

At each bay, it is aligned with the diagonal assembly. Both ends of this cable terminate at a spooler, which uses a motor to reel in the cable, actuating the deployment of the truss.

The nodes perform a number of functions: they contain pulleys to route the drive cable, they provide interfaces to the spokes (at each node, four spokes are attached, two near the top of the node, and two near the bottom), and they contain synchronization gears that mechanically link adjacent bays. These gears ensure that the actuation of one bay causes adjacent bays to also actuate, thus synchronizing all bays during deployment. The synchronization gears disengage in the final $\sim 10 \%$ of deployment. This is because the drive mechanism is sized to drive bays to full deployment one-at-a-time, as opposed to driving bays to full deployment synchronously.

The petal interfaces are bonded to the longerons. In flight, each longeron would have three petal interfaces bonded to it, one in the middle and two at the ends. It was necessary to remove one of the end petal interfaces from each longeron during the process of modifying the truss. (A pre-existing perimeter truss was utilized for this experiment. It was modified by constructing new, wider nodes to better match the SRM deployed and stowed diameters.) As such, in this experiment, each longeron has two petal interfaces: one in the middle and one at the end, as shown in Fig. 6.

In flight, hinges at the base of a petal would be attached to the three petal interfaces. For the present experiment, precision parts that stood in for the petal hinges were used instead. These petal hinge stand-ins were shimmed, as the flight petals would be, and their locations were measured after each deployment using metrology targets affixed to them. For a flight article, petal position error as a result of non-repeatable unfolding of these hinges is expected to be small: precision revolute hinges for unfoldable spacecraft structures have been demonstrated with micron-level repeatability. ${ }^{24-26}$ Future tests will include these hinges and quantify their effect on petal position error.

The longerons and shorterons were off-the-shelf square-cross-section CFRP tubes. The nodes were assembled from off-the-shelf CFRP plates and a central aluminum I-beam. The nodes were bonded together using room-temperature-cure epoxy.

\subsubsection{Spokes}

The spokes are pulled into tension when deployed. This preload is critical in creating a stiff and accurate deployed inner disk structure. Ensuring uniform spoke tension, uniform spoke length under the nominal preload, and uniform spoke stiffness is important for deployment accuracy. Four spokes attached to every perimeter truss node, for a total of 112 spokes. The nominal spoke preload in the deployed state is $71 \mathrm{~N}$. Figure 8 shows the lacing pattern used for this testbed.

The 5.2-m-long spokes are comprised of a unidirectional CFRP tape (Hexcel ${ }^{\circledR}$ IM7 carbon fibers in a polyether ketone ketone matrix) $6.35-\mathrm{mm}$ wide and about $0.10-\mathrm{mm}$ thick. At either end of each spoke, metal end-tabs are bonded using polyetherimide resin to the CFRP tape. These metal end-tabs provide interfaces to the structural hub on one end and to the perimeter truss on the other end of the spoke. The spoke CFRP tape is protected by a flexible braided polyether ether ketone sheath.

This spoke construction is for low coefficient of thermal expansion (CTE) - measured to be $-0.33 \mathrm{ppm} / \operatorname{deg} \mathrm{C}$ at $20^{\circ} \mathrm{C}$. This low-spoke CTE is desirable for the in-space thermal stability of 


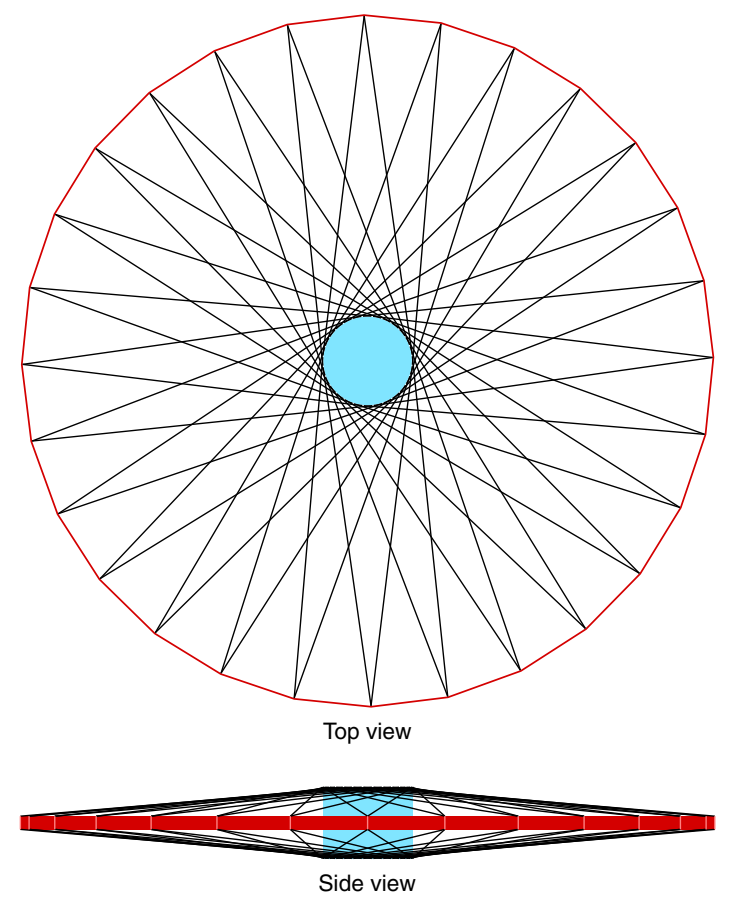

Fig. 8 Spoke lacing pattern. Four spokes are attached to each perimeter truss node, two at the top and two at the bottom. The spokes are attached to the hub such that the tensioned spoke is tangential to the hub cylinder. At each node, the two top spokes are routed to the top of the hub, and the two bottom spokes are routed to the bottom of the hub.

the deployed inner disk. Reference 27 described the thermal stability performance of the inner disk and included details about the in-space thermal environment to which the deployed inner disk is subject.

The spokes were manufactured using a custom-built jig. This jig holds the CFRP tape in a horizontal orientation at the nominal tension of $71 \mathrm{~N}$, while the metal end-tabs are bonded to the CFRP tape. A precision Invar bar 5.2-m in length was used to provide precise separation between the ends of the spoke. As manufactured, the average spoke length at the nominal tension was $5209.616 \mathrm{~mm}$ with a standard deviation of $0.054 \mathrm{~mm}$. The average spoke stiffness EA at the nominal tension was $90.13 \mathrm{kN}$ with a standard deviation of $8.09 \mathrm{kN}$. This low spread in spoke length and spoke stiffness is beneficial in obtaining an inner disk that deploys sufficiently repeatably.

\subsubsection{Structural hub}

As shown in Fig. 9, the structural hub consists of a central cylinder, two spoke rings, and two truss flanges. Each spoke ring is a precision part to which 56 spoke interfaces are attached. The spoke rings were bonded to the central cylinder, after which the location of the 112 spoke interfaces was shimmed to minimize spoke-to-spoke variation in deployed length (within $\pm 200 \mu \mathrm{m}$ of the mean manufactured spoke length assuming an ideal perimeter truss).

A more flight-like hub would be constructed of CFRP materials (for thermal stability and specific stiffness) compared to the aluminum used here. Additionally, a more flight-like hub would include launch-restraint latches to hold the perimeter truss in its stowed position. However, these latches were not included in the present hub. These latches are not critical to the inner disk deployment accuracy: there is a low likelihood of dynamics during the unlatching and initial deployment of the inner disk (it is in self-equilibrium when stowed), and the deployed shape of the inner disk is set only in the final stages of deployment (as supported by data shown in Sec. 7.3).

The hub sits on of a fixture that restrains all translational degrees of freedom and rotational degrees of freedom about the $x$ and $y$ axes, but allows free motion about the $z$ axis. This fixture is further described in Sec. 3.2. 


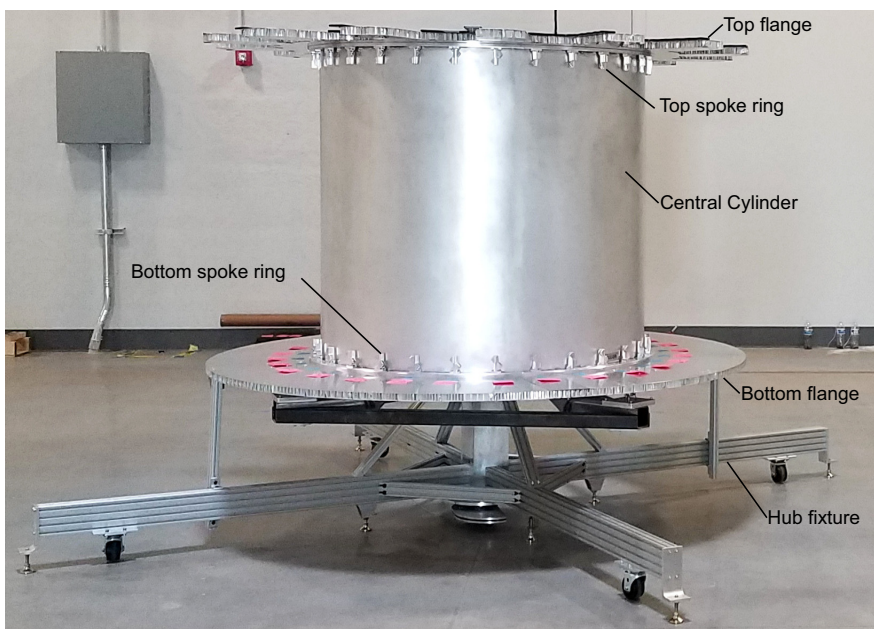

Fig. 9 The structural hub and the hub fixture.

\subsubsection{Optical shield}

The optical shield is a multi-layer structure that makes the inner disk opaque. As shown in Fig. 10, its global shape is a right circular cone with an opening angle of $169 \mathrm{deg}$, with an outer diameter of $10.5 \mathrm{~m}$, and a 1.5 -m-diameter inner cutout where it connects to the central hub. Stowed, the optical shield is $2.2-\mathrm{m}$ in diameter.

The optical shield consists of a number of planar panels hinged together with revolute joints. It can be be folded into a compact configuration for launch. The placement of the hinges (i.e., the fold pattern) was designed using a modification of an existing origami-pattern-generation algorithm. ${ }^{28,29}$ This modified algorithm generates fold patterns that enable conical surfaces to wrap compactly while accounting for panel thickness in the folded configuration. This algorithm also guarantees that the fold lengths are the same in the deployed and the stowed configurations, i.e., that the structure is strain-free when deployed and stowed.

Figure 10 shows the fold pattern, deployed and stowed. The fold pattern consists of 28 major folds: 14 major valley folds (dark red in Fig. 10) and 14 major mountain folds (dark blue in Fig. 10). The major fold lines fold nearly $180 \mathrm{deg}$. There are also a number of minor fold lines (shown as the light lines in Fig. 10) that fold nearly $360 \mathrm{deg} / 28=12.86 \mathrm{deg}$.

Figure 11 shows the construction of the optical shield. The panels, which are triangular or quadrilateral in shape, consist of an aluminum frame along the perimeter to which a 16-mm-thick

(a)

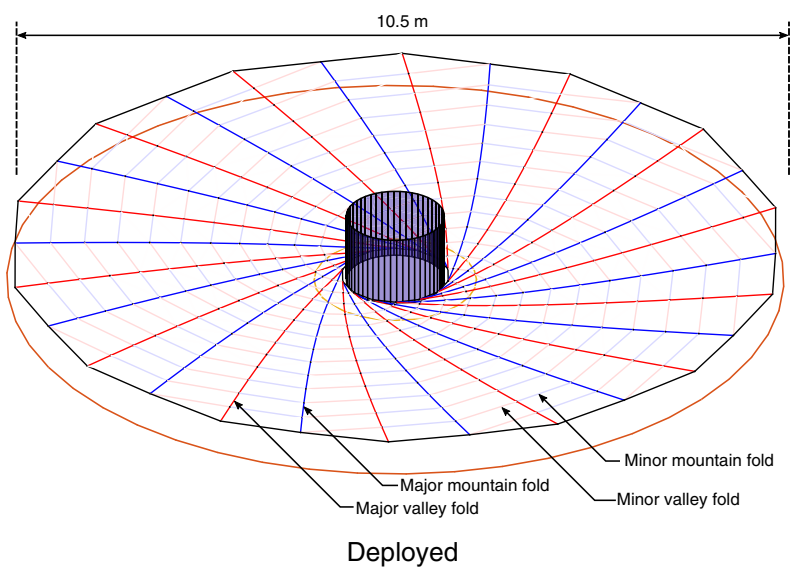

(b)

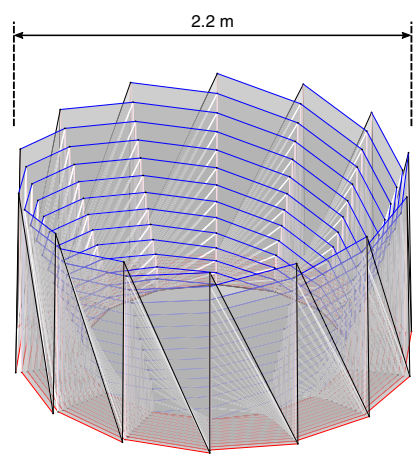

Stowed* $^{*}$

Fig. 10 Isometric views of the optical shield fold pattern: (a) deployed and (b) stowed. Deployed, the optical shield is conical; stowed, it consists of spiral wraps that account for the panel thickness. 


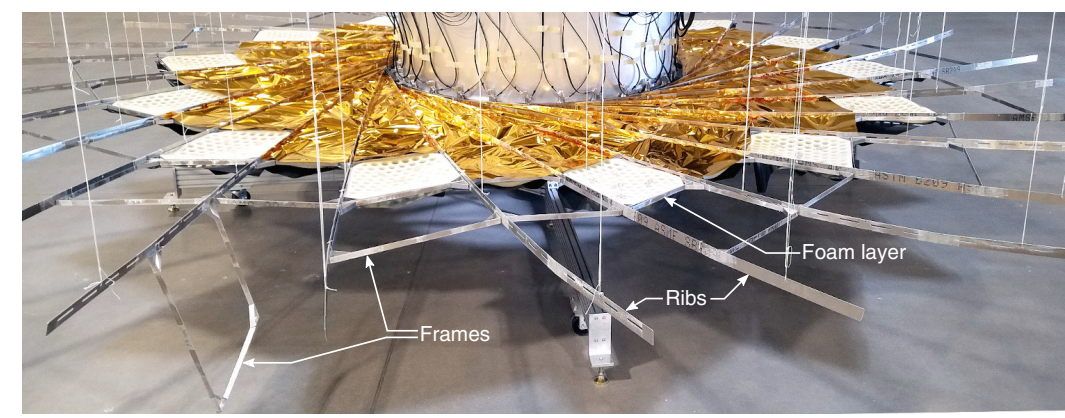

Fig. 11 The internal structure of the optical shield during construction. A number of aluminum frames can be seen without integrated blankets. The ribs extend beyond the shown frame network. The construction of the blankets can also be seen.

blanket is attached. The aluminum frame for each quadrilateral panel is made of four beams 16$\mathrm{mm}$ tall and 1-mm thick. The blanket consists of two layers of $25-\mu \mathrm{m}$-thick Kapton separated by a layer of 16-mm-thick polyurethane foam. The layer of polyurethane foam was lightweighted by cutting out a hexagonal grid of circles from the foam. The separation between the two layers of the blanket mitigates the effect of micrometeoroids: the vast majority of micrometeroids will produce holes in the two layers that are misaligned with the telescope boresight. This avoids a "straight shot" light leak from the star to the telescope.

As shown in Fig. 11, aluminum ribs were placed along all major fold lines. These ribs were constructed from aluminum bars, 1-mm thick and 32-mm tall, hinged together at discrete locations. The frames and the ribs - the internal framework of the optical shield-increase the out-ofplane bending stiffness greatly, which aids gravity offloading. Section 3.2 describes the gravity compensation systems in detail.

The innermost ring of the optical shield was pinned to the hub. The outermost ring of the optical shield was attached to the perimeter truss nodes using lengths of cable, which are slack in the deployed configuration. Thus the deployed optical shield is structurally decoupled from the perimeter truss. This decoupling is necessary for the deployment repeatability of the perimeter truss.

\section{Test Apparatus}

\subsection{Petal Furling Fixture}

A fixture (see Fig. 12) was used to furl the petal test article to a diameter of $2.3 \mathrm{~m}$, which is the outer diameter of the stowed perimeter truss around which petals would be wrapped for flight. In this fixture, a wood drum simulates the stowed perimeter truss. The base of the petal test article is attached to the drum using three hinges, similar to the petal attachment to the perimeter truss in the flight design. Three straps, attached to the drum at one end and tensioned using hanging weights at the other, constrain the petal test article to wrap around the drum as the petal test article is slid off a flat table. Human operators rotate the drum, ensuring slow and quasi-static furling and deployment.

\subsection{Gravity Compensation for the Inner Disk}

To simulate the in-space deployment of the inner disk test article, gravity offloading was used. The inner disk test article was gravity offloaded at 140 discrete locations: lines were attached to the structure at these points, routed over low-friction pulleys, and attached to counterweights. These low-friction pulleys were affixed to wheeled carts free to move along 28 overhead rails, as shown in Fig. 4. Gravity offload was present throughout deployment.

The rail-and-cart system was $\sim 5 \mathrm{~m}$ above the perimeter truss. This height leverages smallangle effects to reduce spurious off-vertical loads on the structure due off-vertical offload lines. 


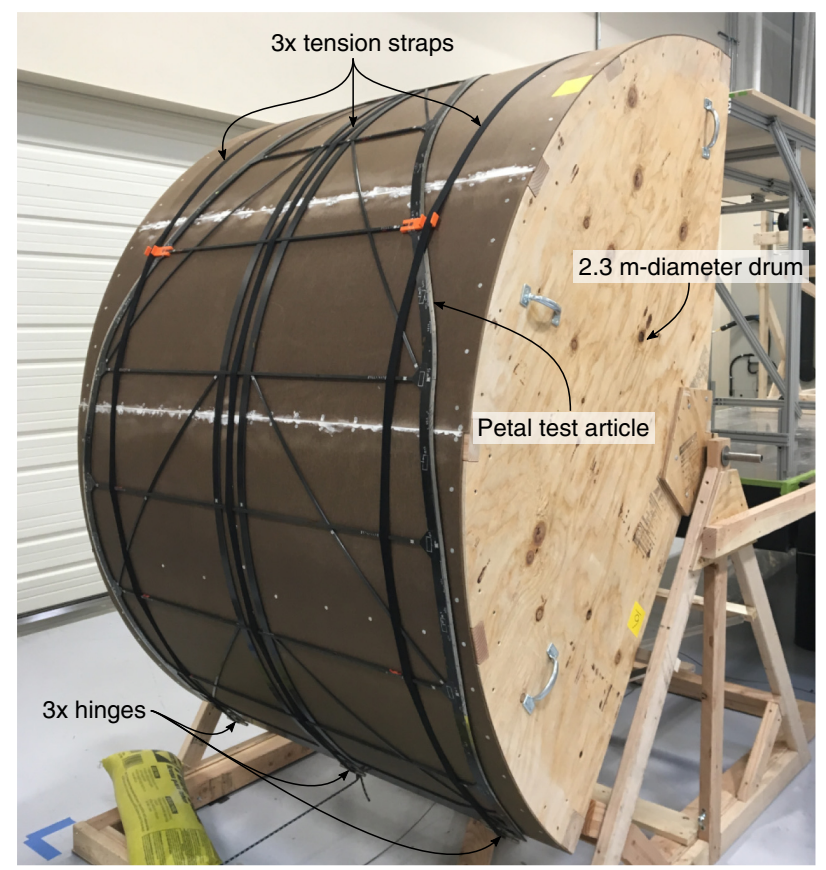

Fig. 12 Petal test article furled on the fixture.

As shown in Fig. 4, four offload points were used for each optical shield major fold line. The locations of these points were chosen to minimize deployed out-of-plane optical shield deflections. One offload point was used for each perimeter truss node.

The structural hub was held by a fixture that restrained, relative to the ground, all translational degrees of freedom and the rotational degrees of freedom about the $x$ and $y$ axes. The rotational degree of freedom about the $z$ axis was left free as the hub must rotate relative to the perimeter truss during deployment as the optical shield is unwrapped. The hub fixture is shown in Fig. 9.

\subsection{Metrology}

\subsubsection{MicroVu}

A MicroVu Excel 250ULC machine was used to measure the width profile of the petal test article. The MicroVu machine consists of a digital microscope mounted on an $(x, y)$-translation stage. MicroVu software detects the 2D location of edges of test articles from microscope images using a contrast-based algorithm. Linear encoders on the two orthogonal axes of the translation stage register microscope images in a global machine coordinate system. MicroVu software outputs the measured 2D edge locations in this global coordinate system.

The size of the MicroVu bed is $2.5 \mathrm{~m} \times 1.6 \mathrm{~m}$. This accommodates the $1.5-\mathrm{m}$ width of the petal test article; however, the 3.8-m length of the petal test article requires two separate scans to measure. The "base" scan measures from the base of the petal test article to the seventh batten (counting from the base), and the "tip" scan measures from the seventh batten to the tip.

During measurements, the petal test article was oriented parallel to the ground and rested on a number of low-friction support blocks. These low-friction blocks apply upward forces to locally react the weight of the test article while minimizing in-plane loads. The test article is free to locally lift up off these blocks. This is a crude form of gravity compensation. A more elaborate method of gravity compensation was not used due to the insensitivity of the starshade contrast metric to out-of-plane petal deformations (e.g., bending and twisting), which have second-order effects on projected in-plane petal shape. Future testing will quantify petal out-of-plane shape repeatability.

To combine data from the base and tip scans, a common coordinate system was established using four cross-hair targets bonded to battens 4, 5, 6, and 7 along the centerline of the petal. 


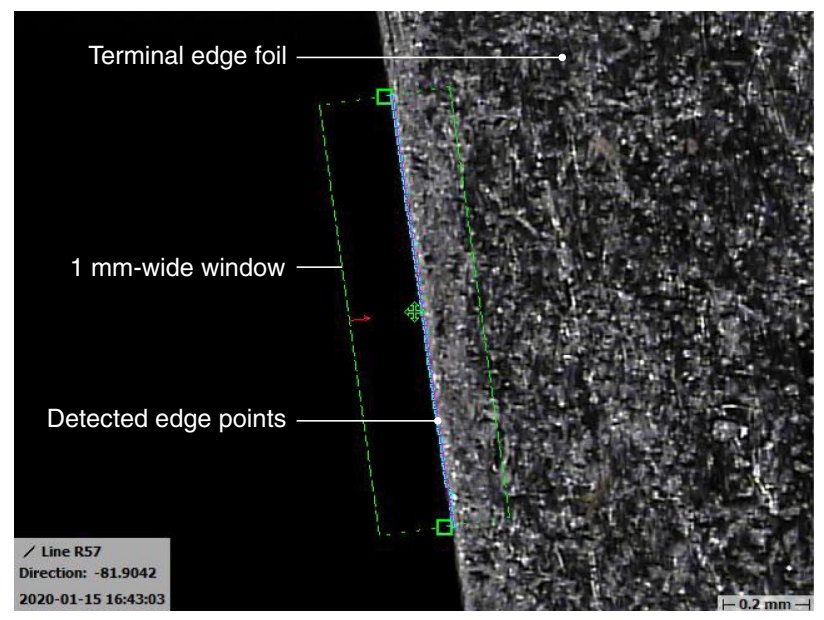

Fig. $131-\mathrm{mm}$-wide MicroVu measurement window of the terminal edge of the petal test article, containing $\sim 100$ detected edge points.

These cross-hair targets are captured in both the base and the tip scans. A best-fit line through the centers of these four targets defines the local $x$ axis and the perpendicular $y$ axis.

The MicroVu was programmed to measure each edge of the petal test article using 185 1mm-wide "windows" spaced $2 \mathrm{~cm}$ apart, with each window containing $\sim 100$ detected edge points. A single-measurement window is shown in Fig. 13, with the detected edge points in blue.

There are two sources of measurement uncertainty in this system: repeatability errors (e.g., due to variations in the edge-detection algorithm, in microscope pointing from structural compliance of the machine, etc.), and thermal strain of the linear encoders (the MicroVu machine does not measure its internal temperature and does not automatically compensate for this effect). The MicroVu Excel 250ULC has a stated length measurement accuracy of $\pm(5.5+L / 300) \mu \mathrm{m}$, where $L$ is the length being measured in millimeters. ${ }^{30}$ The length-independent term $(5.5 \mu \mathrm{m})$ accounts for repeatability errors, and the length-dependent term $(L / 300 \mu \mathrm{m})$ accounts for linear encoder thermal strain effects.

For the petal test article, which has large dimensions along both axes, the linear encoder thermal strain effects dominate measurement noise. Each linear encoder is a ruled gold-plated steel tape that is bonded to a substrate; along the $x$ axis the substrate is a granite bench, and along the $y$ axis the substrate is a steel gantry. Variations in substrate temperature and substrate thermal strain result in thermal strain of the linear encoders. Linear encoder thermal strain results in length measurement error since the MicroVu uses these encoders as length references. The errors along the $x$ and $y$ axes are orthogonal.

To correct measured data for these errors, the MicroVu was instrumented with six high-accuracy $\left( \pm 0.15^{\circ} \mathrm{C}\right.$ absolute, $\pm 0.01^{\circ} \mathrm{C}$ random error) resistance temperature detectors (RTDs), one on each corner of the granite bench (to which the $x$ axis encoder is bonded), and two on each end of the steel gantry (to which the $y$ axis encoder is mounted). Machine temperatures were measured contemporaneously with scans of the petal test article. The CTE of the $x$ and $y$ linear encoders were experimentally measured using -CTE truth bars as reference lengths while the machine temperature was varied. The CTE of the $x$ axis encoder was determined to be $4.9 \mathrm{ppm} /$ $\operatorname{degC}$, and the CTE of the $y$ axis encoder was determined to be $11.2 \mathrm{ppm} / \mathrm{degC}$. These measured CTEs agree with literature-reported values for granite and steel. ${ }^{31,32}$ Section 5.1 describes the methods used to correct the measured data for these linear encoder thermal strain effects.

\subsubsection{Leica laser tracker}

A Leica AT402 laser tracker ${ }^{33,34}$ was used to measure the deployed shape of the inner disk test article. A number of spherically mounted retroreflectors (SMRs) were affixed to the test article. The AT402 laser tracker comprises an absolute distance meter (ADM) mounted on an azimuth/ elevation rotary stage. The ADM measures the range to the SMR and two angular encoders on 
the rotary stage measure azimuth and elevation. Thus the laser tracker can measure the 3D location of the center of an SMR. An SMR consists of a hollow corner-cube retroflector mounted in a 12.7-mm-diameter steel sphere, such that the apex of the corner cube is coincident with the center of the sphere. The laser tracker also has an integrated weather station that records air temperature and relative humidity contemporaneous with each measurement. Spatial Analyzer $^{\circledR}$ metrology software was used to command and gather measurements from the laser tracker.

Laser trackers have sufficiently small measurement uncertainties for this experiment. The stated maximum permissible error of the AT402 laser tracker is $\pm(15 \mu \mathrm{m}+6 \mu \mathrm{m} / \mathrm{m}$ of measurement range).$^{33}$ In practice, however, the laser tracker software reports an uncertainty for each measurement, and the $3 \sigma$ uncertainty was approximately half the maximum permissible error. These reported uncertainties are calculated based on the spread of 1000 independent readings of the 3D location of an SMR. The laser tracker software automatically collects these 1000 readings for each individual measurement.

The laser tracker was placed $\sim 1.5 \mathrm{~m}$ away from the edge of the deployed perimeter truss and elevated $\sim 3.2 \mathrm{~m}$ above the ground. The elevation allowed for a clear view of the SMRs on the far side of the truss, unobscured by the hub.

Of the SMRs affixed to the inner disk test article, the laser tracker could reliably see 34 attached to the petal interfaces on the perimeter truss longerons: 22 attached to the middle petal interfaces and 12 attached to the end petal interfaces. A number of petal interfaces locations were obscured by test hardware. Despite this, there were no large gaps between measured petal interface locations on the perimeter truss: 25 of the 28 longerons had at least one of their petal interface locations measured, and 9 of the 28 longerons had both petal interface locations measured. Additionally, four SMRs were affixed to the floors and the walls of the room as drift markers.

\section{Test Procedures}

\subsection{Petal Furling and Measurement}

To establish a baseline, the width profile of the petal test article was measured using the MicroVu prior to furling. Then the petal test article was furled and deployed 5 times using the furling fixture. After, the width profile of the petal test article was measured.

To mitigate the effect of the thermal strain of the MicroVu encoders, petal width profile measurements were taken only when the MicroVu internal temperatures were close to the internal temperatures observed during the baseline measurement (within $\pm 1^{\circ} \mathrm{C}$ for the granite bench and within $\pm 0.6^{\circ} \mathrm{C}$ for the steel gantry). Additionally, low-CTE $(0.13 \mathrm{ppm} / \mathrm{degC}) \mathrm{CFRP}$ rods were used as "truth bars" to verify MicroVu measurement repeatability. The lengths of these rods measured alongside the petal test article were within $\pm 10 \mu \mathrm{m}$ of their measured baseline lengths.

\subsection{Inner Disk Shimming and Deployment}

Fifty-eight deployments of the inner disk test article were conducted. About 10 of these were for initial verification of functionality, and 26 of these were to conduct shimming of the petal interfaces. The remaining 22 deployments were performed in the final shim state.

For a flight starshade, the locations of the petal hinges would be shimmed to reduce static petal position errors. In this experiment, the SMR locations were shimmed as the petal hinges would be. The radial and tangential location of each SMR attached to a petal interface was adjusted by placing metal shims between the SMR mount and the petal interface bonded to the longeron.

Eight rounds of measurement and shim adjustment were performed. For each round of measurement, at least three deployments were conducted, establishing a mean deployed position for each SMR. Based on these measured deployed locations, shim corrections were computed and implemented. The decision to stop after eight rounds of shimming was based on schedule, Additional shimming rounds could have been performed to reduce shape errors. 

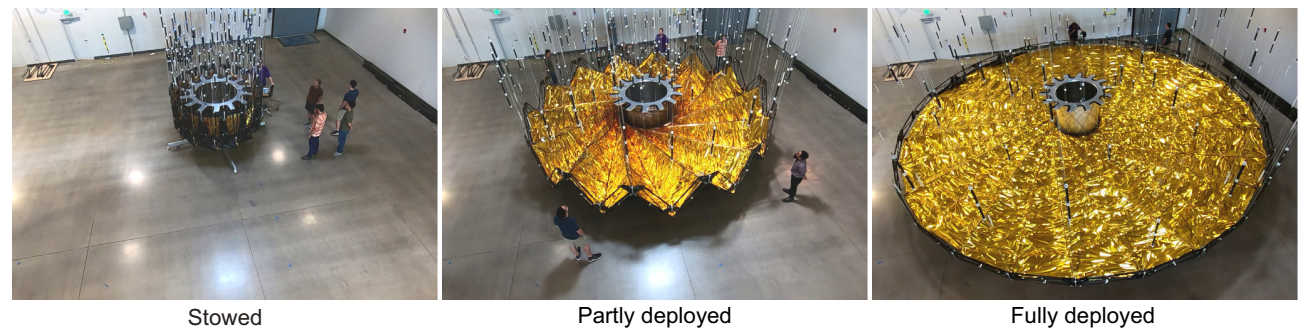

Fig. 14 Deployment from a 96\% stowed state. The images are at the same scale.

The shimming process emphasized the reduction in petal translation errors. In shimming the SMR positions, clocking static errors were not controlled. Despite this, clocking accuracy performance is estimated in Sec. 5.2 by considering the measured clocking repeatability and the magnitude of expected clocking shimming errors.

Twenty-two deployments were performed with the final shim state. A number of these deployments started from a partially stowed state. This was to save time: a full stow of the inner disk test article takes about $5 \mathrm{~h}$, whereas a $8 \%$ partial stow takes only a half an hour. It was expected that partial deployments would be representative of full deployments in terms of deployment repeatability since the final deployed position of the inner disk is set only in the final few percent of deployment. This expectation was confirmed by the measured data, as discussed in Sec. 7.3.

The degree of stowage is quantified as a stow percent $s$, which is defined as the angle between the longerons when stowed, divided by $180 \mathrm{deg}$, which is the angle between the longerons when fully stowed:

$$
s=\frac{\theta_{\text {longerons }}}{180 \text { deg }} \times 100 \% \text {. }
$$

Thus $s=0 \%$ is fully deployed and $s=100 \%$ is fully stowed. Of the 22 deployments performed in the final shim state, 11 were from $8 \%$ stowed, 3 from $49 \%$ stowed, 3 from $82 \%$ stowed, and 5 from $96 \%$ stowed.

The inner disk test article was not stowed to $100 \%$ since the hub did not have the requisite launch-restraint latches to capture the fully stowed truss. However, as shown in Sec. 7.3, the deployment accuracy of the inner disk test article does not change with the degree of stowage.

Deployments from the $96 \%$ stow state took about $25 \mathrm{~min}$. This slow, quasi-static deployment rate was chosen to reduce the air drag effects and is representative of expected in-space deployment. Figure 14 shows three stills from a video of a deployment from a $96 \%$ stow state.

\section{Data Processing and Analysis}

\subsection{Petal Width Change}

The petal shape is characterized by its width profile $w(x)$, the distance along the $y$ axis between the $+y$ and the $-y$ terminal edges as a function of longitudinal position $x$ (see Fig. 2 for the definition of the petal coordinate system). This experiment is concerned with the change in width profile due to stowage-and-deployment cycles: $\Delta w(x)=w_{1}(x)-w_{0}(x)$, where $w_{0}(x)$ is the width profile at the baseline condition and $w_{1}(x)$ is the width profile after stowage-and-deployment cycles.

As described in Sec. 3.3.1, the MicroVu was programmed to follow each edge and capture 185 1-mm-wide windows for each edge. The MicroVu outputs the 2D location of $\sim 100$ points in each window. These points are used to evaluate the petal width at each window. The final width profile is evaluated at these $185 x$ locations, roughly corresponding to the centers of the measurement windows.

The MicroVu measurement outputs are corrected for errors due to the thermal strain of the $x$ and $y$ linear encoders (discussed in Sec. 3.3.1). The $x$ and $y$ encoder temperatures were measured 
contemporaneously with the petal scans, and thus the temperature changes $\Delta T_{x}$ and $\Delta T_{y}$ relative to the baseline are known.

Since the window positions along the $x$ axis are hard-coded in the MicroVu program, the actual measured window positions vary from scan to scan due to machine repeatability errors or $x$ axis thermal strain. For example, consider a measurement taken at $\Delta T_{x}>0$. The microscope head is commanded to a hard-coded $x$ location, but since the $x$ linear encoder has expanded, the microscope travels farther than it did during the baseline scan.

To compare petal width measurements at the same physical location, a corrected location $x_{\mathrm{cor}, i}$ is computed, where $i$ is the window index, an integer between 1 and 185. The corrected $x$ location is a function of reference $x$ positions $x_{\text {ref }, i}$, which are the window centers for the $-y$ edge in the baseline scan, and the thermal strain of the $x$ axis:

$$
x_{\mathrm{cor}, i}=\frac{x_{\mathrm{ref}, i}}{1+\mathrm{CTE}_{x} \Delta T_{x}},
$$

where $\mathrm{CTE}_{x}=4.9 \mathrm{ppm} / \operatorname{deg} \mathrm{C}$ is the measured CTE of the $x$ axis linear encoder.

For each window and corrected $x$ position $x_{\mathrm{cor}, i}$, the $y$ position of the edge $y_{i}$ is calculated using a linear fit through the $\sim 100$ edge points detected in the window:

$$
y_{i}=c_{1, i} x_{\mathrm{cor}, i}+c_{0, i}
$$

where the linear fit coefficients $c_{1, i}$ and $c_{0, i}$ are computed using the $\sim 100$ edge points detected in the window. Across the 1-mm-wide windows, the edge is linear to within $2 \mu \mathrm{m}$.

For each $y_{i}$, a corrected value $y_{\mathrm{cor}, i}$ is calculated to account for the thermal strain of the $y$ linear encoder:

$$
y_{\mathrm{cor}, i}=y_{i}\left(1+\mathrm{CTE}_{y} \Delta T_{y}\right)
$$

where $\mathrm{CTE}_{y}=11.2 \mathrm{ppm} / \operatorname{deg} \mathrm{C}$ is the measured CTE of the $y$ axis linear encoder. This correction has a different form than Eq. (2) since, unlike the $x$ locations of the windows, the $y$ locations of the edge are not hard-coded, but measured with the $y$ axis linear encoder as a length reference.

Note that these corrections account for the thermal deformation of the MicroVu measurement machine only and not the thermal deformation of the petal test article. The thermal deformations of the petal test article over the course of this experiment are expected to be much smaller than those of the MicroVu linear encoders since the width-wise CTE of the petal test article (dominated by the battens with measured CTE of $0.13 \mathrm{ppm} /{ }^{\circ} \mathrm{C}$ ) is much lower than the CTEs of the encoders.

The measured width at $i$ 'th window $w\left(x_{\mathrm{ref}, i}\right)$ is calculated by taking the difference of the corrected $y$ positions of the $+y$ and $-y$ edges. Finally, the petal width change $\Delta w\left(x_{\mathrm{ref}, i}\right)$ at the $i$ 'th window is computed by subtracting the baseline width profile from the width profile measurement after furl-and-deploy cycles.

The driver of instrument contrast degradation due to petal shape change is the petal width strain bias, which is the zeroth-order petal strain over the length of a petal. The petal width strain profile $\varepsilon(x)$ is the change in width relative to width:

$$
\epsilon(x)=\frac{\Delta w(x)}{w(x)} .
$$

In this work, the petal width strain bias is calculated as the median of the petal width strain profile $\epsilon(x)$ over the region $x \in[0 \mathrm{~m}, 2.6 \mathrm{~m}]$. This region excludes the petal tip, which was found to have minimal impact on instrument contrast. Petal width strain bias, calculated thus, was found to correlate well to instrument contrast degradation due to strained petals computed using the boundary integral method. ${ }^{35}$ 


\subsubsection{Petal width change measurement uncertainty}

The stated length measurement accuracy of the MicroVu Excel 250ULC is $(5.5+L / 300) \mu \mathrm{m}$, where $L$ is the measured length in millimeters. ${ }^{30}$ The length-invariant portion of this expression $(5.5 \mu \mathrm{m})$ is attributed to machine repeatability errors, and the length-dependent portion $[(L / 300) \mu \mathrm{m}]$ is attributed primarily to the uncorrected machine thermal strain effects. ${ }^{36}$ Since the data processing method described in Sec. 5.1 largely corrects for the machine thermal strain effects, the measurement uncertainty is estimated using a modified method. The uncertainty is computed as a sum of two components: (1) the machine repeatability errors and (2) the measurement uncertainty due to errors in the correction of the thermal strain effects.

The first component-uncertainty in petal width change due to machine repeatability effects-is taken to be $\sqrt{2} \times 5.5 \mu \mathrm{m}$ since two independent width measurements are subtracted to compute the width change.

The second component-uncertainty due to the correction of the thermal strain effects-is due to uncertainty in measuring the temperature changes $\Delta T_{x}$ and $\Delta T_{y}$ of the $x$ and $y$ axes of the MicroVu, as used in Eqs. (2) and (4). These temperatures were measured using a number of RTD sensors affixed to the MicroVu. The uncertainties in $\Delta T_{x}$ and $\Delta T_{y}$ are computed using the spatial and temporal variations in measured temperatures deltas and were at most $\pm 0.15^{\circ} \mathrm{C}$. The corresponding uncertainty in petal width change was computed by calculating petal width changes at the extremes of the $\Delta T_{x}$ and $\Delta T_{y}$ uncertainty ranges.

\subsection{Inner Disk Shape Error}

Thirty-four SMR targets on the inner disk test article were measured after each of the 22 deployments. The measured location of the SMR at the $i$ 'th petal interface at the $j$ 'th deployment is denoted by $\mathbf{p}_{i j} \in \mathbb{R}^{3} . i$ is an integer between 1 and 34, the total number of measured petal interfaces; and $j$ is an integer between 1 and 22 , the total number of deployments. $\overline{\mathbf{p}}_{i}$ is the mean deployed location (taken over the 22 deployments) of the $i$ 'th petal interface. The nominal petal interface positions are denoted by $\mathbf{p}_{i}^{*}$. These nominal petal interface positions are defined based on a perfect 28-sided polygon centered at the origin and lie in the $x, y$ plane.

There are two measures of shape error that are of interest: accuracy, which is the deviation between the measured locations $\mathbf{p}_{i j}$ and the nominal location $\mathbf{p}_{i}^{*}$; and repeatability, which is the deviation between the measured locations $\mathbf{p}_{i j}$ and the mean deployed location $\overline{\mathbf{p}}_{i}$. Figure 15 illustrates the relation between these values. Both accuracy and repeatability are measures of variation in shape from deployment to deployment. However, accuracy includes a contribution from shimming errors that are secular biases that do not vary between deployments, whereas repeatability is insensitive to these shimming errors. The objective of this activity was to demonstrate that four components of the shape accuracy error-radial bias, radial random, tangential random, and clocking random-fall within allocated bounds.

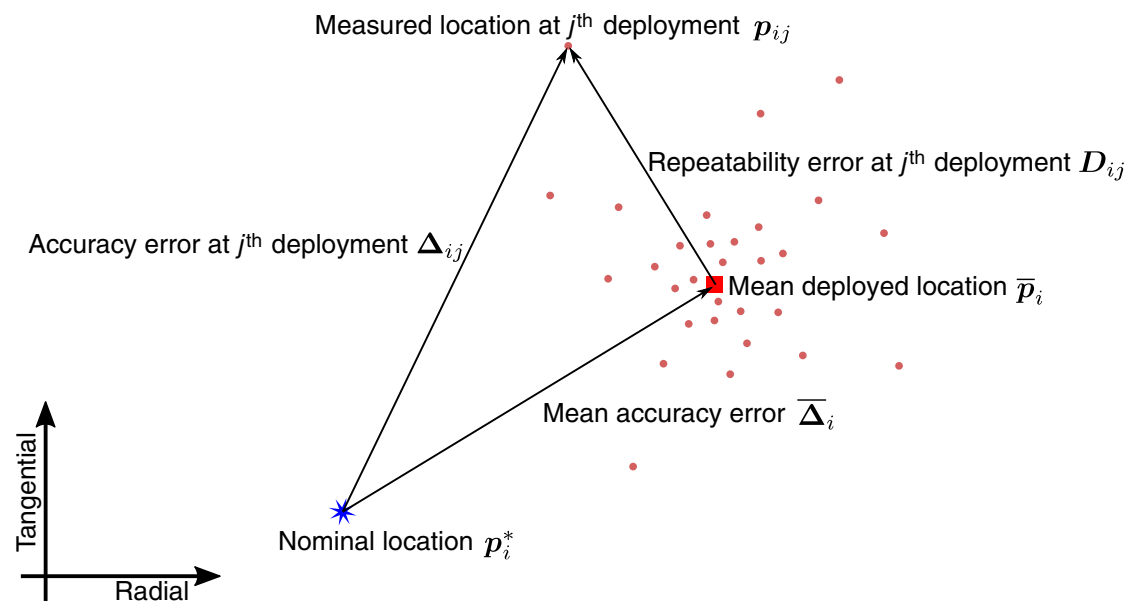

Fig. 15 Definitions of the two measures of inner disk deployment error: accuracy and repeatability. 


\subsubsection{Radial and tangential accuracy}

As shown in Fig. 15, the accuracy error $\Delta_{i j}$ is defined as the difference between the measured location $\mathbf{p}_{i j}$ and the nominal location of the $i$ 'th petal interface $\mathbf{p}_{i}^{*}$ :

$$
\Delta_{i j}=\mathbf{p}_{i j}-\mathbf{p}_{i}^{*}
$$

Of interest are the radial and tangential components of the accuracy error $\Delta_{i j}$. The distribution of these is compared against the radial random and tangential random allocations. The local radial and tangential basis vectors $\hat{\mathbf{r}}_{i}$ and $\hat{\mathbf{t}}_{i}$ for the $i$ 'th petal interface are defined using the nominal location of the petal interface $\mathbf{p}_{i}^{*}$ :

$$
\hat{\mathbf{r}}_{i}=\frac{\mathbf{p}_{i}^{*}}{\left|\mathbf{p}_{i}^{*}\right|} \quad \hat{\mathbf{t}}_{i}=\hat{\mathbf{z}} \times \hat{\mathbf{r}}_{i},
$$

where $\hat{\mathbf{z}}$ is the unit vector along the $z$ axis. Using these local basis vectors, the accuracy error $\Delta_{i j}$ is decomposed into radial and tangential components $\Delta R_{i j}$ and $\Delta T_{i j}$ :

$$
\Delta R_{i j}=\Delta_{i j} \cdot \hat{\mathbf{r}}_{i} \quad \Delta T_{i j}=\Delta_{i j} \cdot \hat{\mathbf{t}}_{i}
$$

The radial and tangential components over the 22 deployments of the $i$ 'th petal interface are fitted with normal distributions:

$$
\Delta R_{i j} \sim \mathcal{N}\left(\overline{\Delta R}_{i}, \sigma_{R i}^{2}\right) \quad \Delta T_{i j} \sim \mathcal{N}\left(\overline{\Delta T}_{i}, \sigma_{T i}^{2}\right)
$$

where $\overline{\Delta R}_{i}$ and $\overline{\Delta T}_{i}$ are the mean radial and tangential components of the accuracy error of the $i$ 'th petal interface and $\sigma_{R i}$ and $\sigma_{T i}$ are the standard deviations. $\overline{\Delta R}_{i}$ and $\overline{\Delta T}_{i}$ are the in-plane components of the mean accuracy error $\bar{\Delta}_{i}$ defined in Fig. 15, which is indicative of shimming errors.

The standard deviations $\sigma_{R i}$ and $\sigma_{T i}$ are increased to account for the uncertainties in the measured locations. The laser tracker software outputs an uncertainty for each individual measurement. Based on these values, a scalar $1 \sigma$ uncertainty $u_{i}$ can be computed for each measurement $\mathbf{p}_{i j}$. For the present experiment, the $1 \sigma$ uncertainty $u_{i}$ ranges between 1 and $10 \mu \mathrm{m}$ for the petal interfaces. The corrected standard deviations $\hat{\sigma}_{R i}$ and $\hat{\sigma}_{T i}$ are obtained by taking a root-sumsquare of the measured standard deviations and the measurement uncertainties:

$$
\hat{\sigma}_{R i}^{2}=\sigma_{R i}^{2}+u_{i}^{2} \quad \hat{\sigma}_{T i}^{2}=\sigma_{T i}^{2}+u_{i}^{2} .
$$

Given the low sample size-22 deployments in total - the standard deviations of the sample may differ from the standard deviations of the underlying population. To address this uncertainty, tolerance intervals are employed, following the process set out by Ref. 17. A tolerance interval is $\mathrm{a} \pm k \sigma$ region centered around the mean that will contain a fraction $\gamma$ of future members of a population with a confidence level defined by $(1-\alpha) . \sigma$ is the sample standard deviation, and $k$ is a factor dependent on the number of deployments $n, \alpha$, and $\gamma$. Computing $k$ exactly is challenging. A conservative estimate for $k$ is used in Refs. 17 and 37:

$$
k=\sqrt{\frac{(n-1) \chi_{1, \gamma}(1 / n)}{\chi_{n-1, \alpha}}},
$$

where $\chi_{1, \gamma}(1 / n)$ is a quantile from a non-central chi-square distribution, and $\chi_{n-1, \alpha}$ is a quantile from a standard chi-square distribution. A tolerance interval is used that will contain $99.73 \%$ of future members with $90 \%$ confidence, i.e., $\gamma=0.9973$ and $(1-\alpha)=0.90 . n=22$ gives $k=$ 3.8596 and a tolerance interval of $\pm 3.8596 \sigma$. This is a conservative estimate for the $\pm 3 \sigma$ region around the mean for a well-sampled normal distribution, which contains $99.73 \%$ of the population. 


\subsubsection{Deployment repeatability}

Based on Fig. 15, the repeatability error $\mathbf{D}_{i j}$ is defined as the difference between the deployed position after the $j$ 'th deployment $\mathbf{p}_{i j}$ and the mean deployed position over all deployments $\overline{\mathbf{p}_{i}}$ :

$$
\mathbf{D}_{i j}=\mathbf{p}_{i j}-\overline{\mathbf{p}}_{i} .
$$

As defined in Fig. 15, the accuracy error $\Delta_{i j}$ is the sum of the repeatability error $\mathbf{D}_{i j}$ and the mean accuracy error $\bar{\Delta}_{i}$ :

$$
\Delta_{i j}=\mathbf{D}_{i j}+\bar{\Delta}_{i}
$$

Thus the accuracy error $\Delta_{i j}$ arises from deployment repeatability errors $\mathbf{D}_{i j}$ that change from deployment to deployment and a mean accuracy error $\bar{\Delta}_{i}$ that remains constant. The repeatability error $\mathbf{D}_{i j}$, therefore, is an indication of the deployment accuracy of the structure if the shimming was perfect, i.e., if $\bar{\Delta}_{i}=0$. Now, clearly, perfect shimming is impractical to achieve, but the magnitude of the shimming errors can be reduced.

Taking the standard deviation over all deployments of both sides of Eq. (13), it can be seen that $\sigma\left(\Delta_{i j}\right)=\sigma\left(\mathbf{D}_{i j}\right)$ since the mean accuracy error $\bar{\Delta}_{i}$ is constant over all deployments. This means that the size of the tolerance intervals for the repeatability errors $\mathbf{D}_{i j}$ is the same as calculated above for the accuracy errors $\Delta_{i j}$.

\subsubsection{Radial bias}

Radial bias is the difference between the deployed radius of the perimeter truss and the nominal radius. The radial bias $B_{j}$ at the $j$ 'th deployment can be calculated as the mean of the radial accuracy errors of all the petal interfaces at that deployment:

$$
B_{j}=\frac{1}{34} \sum_{i=1}^{34} \Delta R_{i j}
$$

The 22 measured radial bias terms are fitted with a normal distribution:

$$
B_{j} \sim \mathcal{N}\left(\bar{B}, \sigma_{B}^{2}\right)
$$

where $\bar{B}$ is the mean radial bias across the 22 deployments, and $\sigma_{B}$ is the standard deviation.

As in Sec. 5.2.1, to account for the low sample size of 22 deployments, a tolerance interval of size $\pm k \hat{\sigma}_{B}$ is constructed to place bounds that are equivalent to $3 \sigma$ levels. $k=3.8596$ is obtained using the same inputs as Sec. 5.2.1 $[\gamma=0.9973, n=22,(1-\alpha)=0.90]$.

\subsubsection{Petal clocking}

The in-plane petal clocking accuracy error $\Delta \theta_{i j}$ at the $i$ 'th perimeter truss bay at the $j$ 'th deployment is evaluated as

$$
\Delta \theta_{i j}=a_{x y}\left(\mathbf{p}_{s j}-\mathbf{p}_{t j}, \mathbf{p}_{s}^{*}-\mathbf{p}_{t}^{*}\right)
$$

where $\mathbf{p}_{s j}$ and $\mathbf{p}_{t j}$ are the measured positions of the two petal interfaces at the $i$ 'th truss bay at the $j$ 'th deployment, ()$^{*}$ represents their nominal positions, and $a_{x y}($,$) is a function that returns the$ angle between the projections of its two vector arguments in the $x y$ plane (see Fig. 4 for the coordinate system definition). The measured clocking accuracy errors for each truss bay are fitted with normal distributions:

$$
\Delta \theta_{i j} \sim \mathcal{N}\left(\overline{\Delta \theta}_{i}, \sigma_{\theta i}^{2}\right)
$$


where $\overline{\Delta \theta}_{i}$ is the mean clocking accuracy error and $\sigma_{\theta i}$ is the clocking standard deviation for the $i$ 'th bay over all deployments.

As before, the measured clocking standard deviation is expanded to account for measurement uncertainty:

$$
\hat{\sigma}_{\theta i}^{2}=\sigma_{\theta i}^{2}+u_{\theta i}^{2},
$$

where $u_{\theta i}$ is the $1 \sigma$ measurement uncertainty of the clocking at the $i$ 'th bay. This measurement uncertainty is estimated as

$$
u_{\theta i}=\frac{u_{s}+u_{t}}{\left\|\mathbf{p}_{s}^{*}-\mathbf{p}_{t}^{*}\right\|}
$$

where $u_{s}$ and $u_{t}$ are the scalar uncertainties associated with the measured positions $\mathbf{p}_{s j}$ and $\mathbf{p}_{t j}$.

Since petal clocking errors were not controlled during the shimming process (see Sec. 4.2), absolute clocking accuracy is not representative of a flight-like starshade. Instead, clocking accuracy is estimated as a quadrature sum of measured clocking repeatability and estimated clocking shimming errors. Clocking repeatability $D \theta_{i j}$ for the $i$ 'th perimeter truss bay at the $j$ 'th deployment is evaluated as

$$
D \theta_{i j}=\Delta \theta_{i j}-\overline{\Delta \theta}_{i}
$$

The standard deviations for clocking repeatability are equivalent to those for clocking accuracy since mean clocking error $\overline{\Delta \theta}_{i}$ is constant over all deployments.

As in Sec. 5.2.1, to account for the low sample size of 22 deployments, tolerance intervals $\pm k \hat{\sigma}_{\theta i}$ are constructed to establish $3 \sigma$-equivalent bounds on the spread in clocking repeatability, with $k=3.8596$.

\section{Test Results}

\subsection{Petal Width Change}

Figure 16 shows the measured change in width profile $\Delta w(x)$ of the petal test article from the baseline after five furl-and-deploy cycles. The band around the data represents maximum uncertainty due to the effects discussed in Sec. 5.1.1.

Figure 17 shows the petal width strain profile $\epsilon(x)$ after five furl-and-deploy cycles, using the baseline measurement as the reference. The uncertainty band corresponds to the uncertainty band shown in Fig. 16. The uncertainty in petal strain is higher at the tip because the petal width is smaller.

Based on the petal width strain profile shown in Fig. 17, the petal width strain bias after five furl-and-deploy cycles was calculated to be $-0.55_{-7.30}^{+6.91} \mathrm{ppm}$. As described in Sec. 5.1, the petal width strain bias is the median strain over a certain length of the petal. The uncertainty in petal width strain bias is calculated by evaluating the petal width strain bias for the extrema of uncertainty envelopes of the petal width strain profiles.

\subsection{Inner Disk Deployment Accuracy}

Figure 18 plots the mean radial and tangential components of the accuracy error $\overline{\Delta R}_{i}$ and $\overline{\Delta T}_{i}$, as well as the size of the tolerance intervals around each mean. Additionally, the in-plane components of the accuracy errors $\left(\Delta R_{i j}, \Delta T_{i j}\right)$ for all $i$ and $j$ are shown in Fig. 18. A Monte Carlo (MC) approach, described in Appendix A, was used to determine the $3 \sigma$ levels for the radial and tangential components of the shape accuracy errors across the 34 measured petal interfaces $-121 \mu \mathrm{m}$ radial and $91 \mu \mathrm{m}$ tangential.

Figure 19 plots the radial and tangential components of the repeatability error $\mathbf{D}_{i j}$. All deployment repeatability errors and the associated tolerance intervals fall within $\pm 86 \mu \mathrm{m}$. The error bars indicate the maximum radial and tangential tolerance intervals. 
Arya et al.: Demonstration of deployment repeatability of key subsystems of a furled starshade architecture

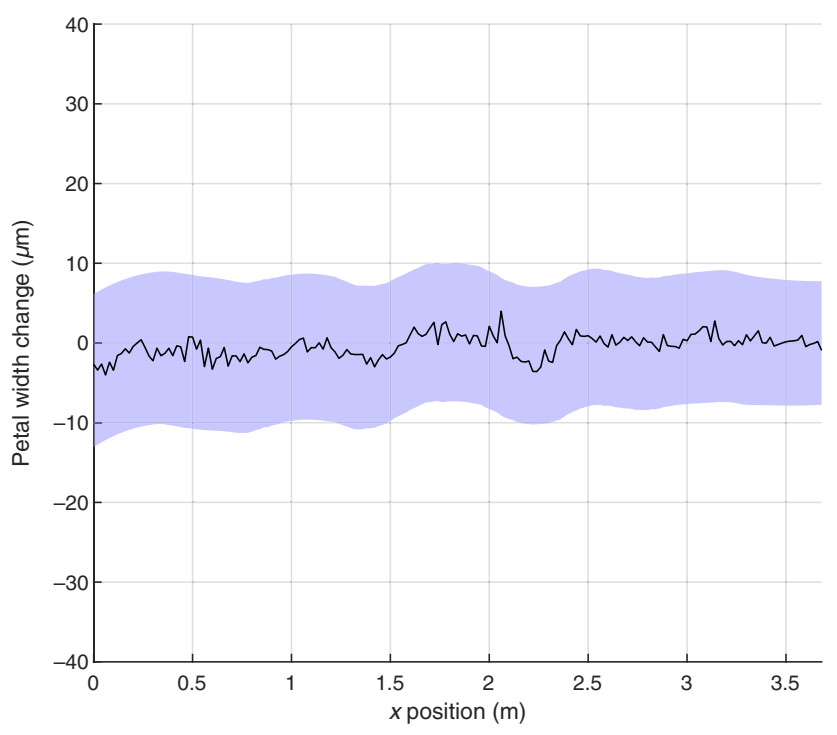

Fig. 16 Petal width change after five furl-and-deploy cycles. The band represents maximum measurement uncertainty.

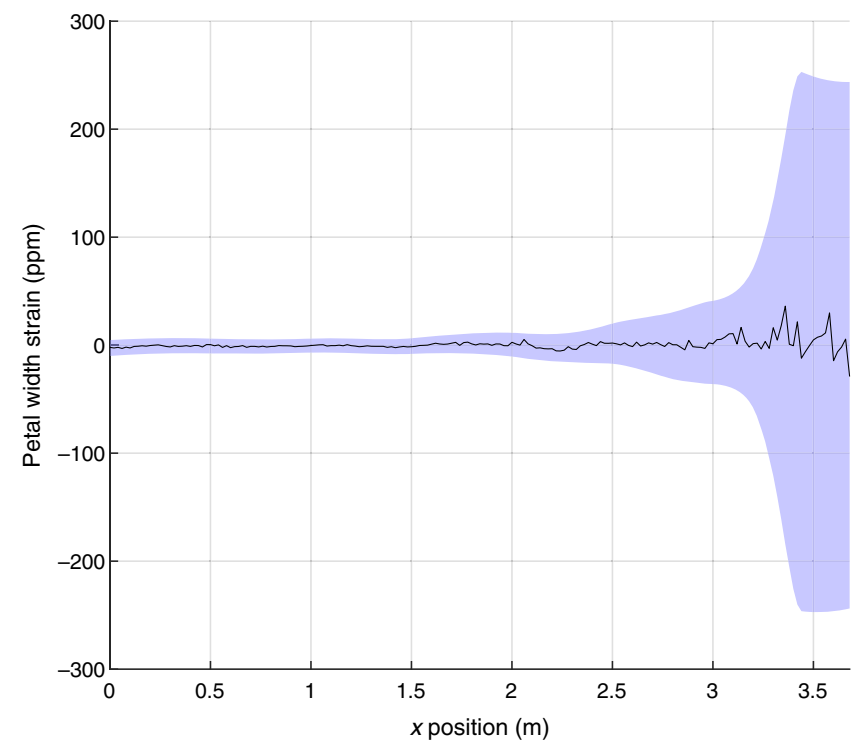

Fig. 17 Petal width strain after five furl-and-deploy cycles. The band represents maximum measurement uncertainty. The uncertainty in strain is higher at the tip of the petal because even though the uncertainty in width change is relatively constant over the length of the petal, the petal width is smaller at the tip, leading to a proportionally higher strain uncertainty.

Figure 20 plots the radial bias for each of the 22 deployments. The $3 \sigma$ uncertainty in each measurement of the radial bias was calculated to be $1.7 \mu \mathrm{m}$ based on the measurement uncertainties $u_{i}$ of the 34 petal interfaces. The standard deviation $\sigma_{B}$ of the measured radial biases is $6.6 \mu \mathrm{m}$. This is expanded to account for the measurement uncertainty, resulting in the corrected standard deviation $\hat{\sigma}_{B}=6.8 \mu \mathrm{m}$. This gives the radial bias tolerance interval as $\pm 26 \mu \mathrm{m}$.

Figure 21 plots the petal clocking repeatability for the 9 perimeter truss bays where clocking was measured, for each of the 22 deployments. The $3 \sigma$-equivalent tolerance intervals for each of the 9 bays are also shown. The measured $3 \sigma$ clocking repeatability is $105 \mu \mathrm{rad}$. This is the quadrature sum of the tolerances intervals associated with the 9 bays. These tolerance intervals account for the clocking measurement uncertainty, which was between 11 and $15 \mu \mathrm{rad}$ for the 9 bays. 
Arya et al.: Demonstration of deployment repeatability of key subsystems of a furled starshade architecture

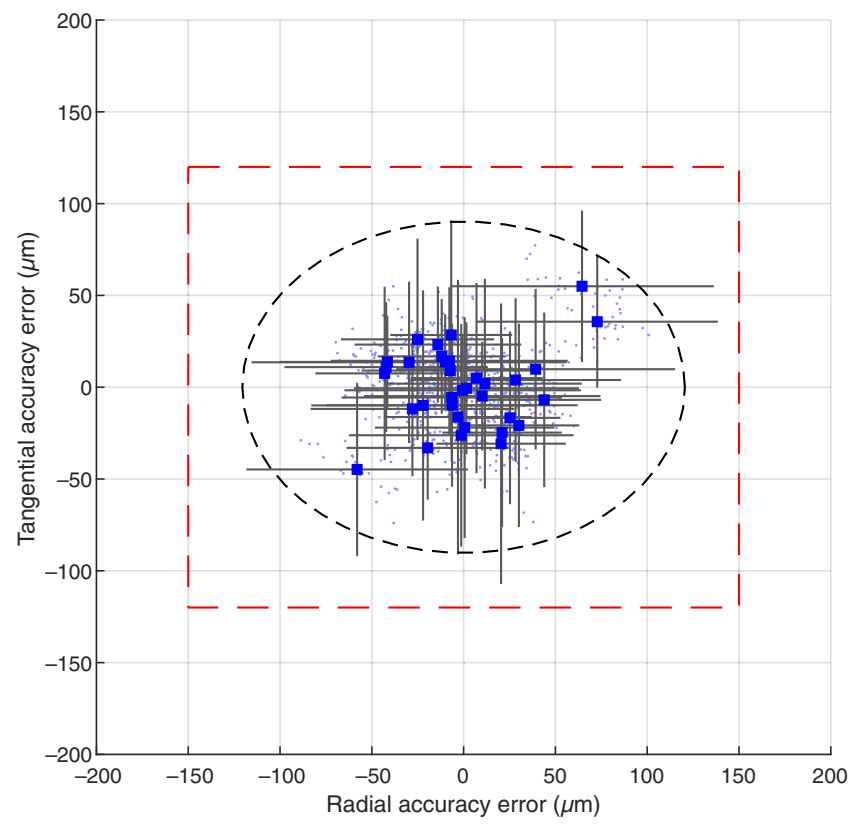

Fig. 18 Radial and tangential components of the measured deployment accuracy errors of the petal interfaces. The blue squares represent the mean accuracy errors of the measured petal interfaces, and the bars represent the tolerance intervals around the mean. Accuracy errors of the petal interfaces from individual deployments are shown as light blue dots. The red rectangle represents the radial $(150 \mu \mathrm{m})$ and tangential $(120 \mu \mathrm{m})$ accuracy allocations. The major and minor axes of black dashed ellipse represents the $3 \sigma$ radial and tangential accuracy errors $-121 \mu \mathrm{m}$ radial and $91 \mu \mathrm{m}$ tangential-calculated using the $\mathrm{MC}$ analysis described in Appendix A.

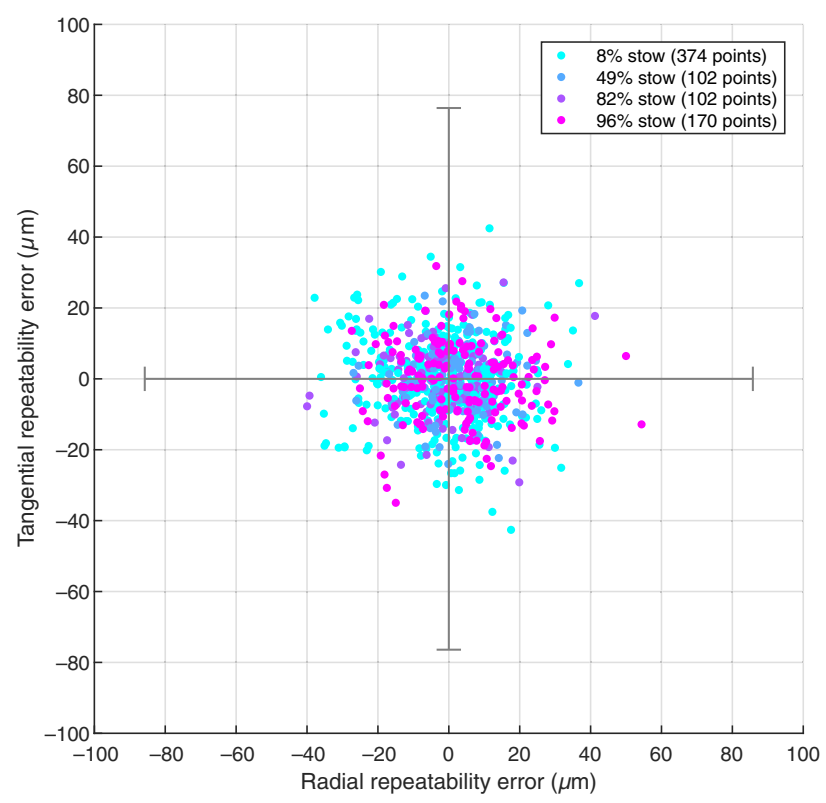

Fig. 19 In-plane components of the deployment repeatability errors of the 34 measured petal interfaces over all 22 deployments. The bars represent the maximum radial and tangential tolerance intervals across the 34 petal interfaces. The color corresponds to the stow percent associated with the deployment. Since a different number of deployments was performed for each stow percent, there is a different number of points for each stow percent.

To estimate clocking accuracy, the measured repeatability is quadrature summed with the expected clocking shimming errors. The clocking shimming error at a truss bay arises entirely from the radial shimming errors of the two petal interfaces at that truss bay. As such, given the measured $3 \sigma$ radial shimming error $e_{R}=89 \mu \mathrm{m}$ (calculated from the spread of mean radial 
Arya et al.: Demonstration of deployment repeatability of key subsystems of a furled starshade architecture

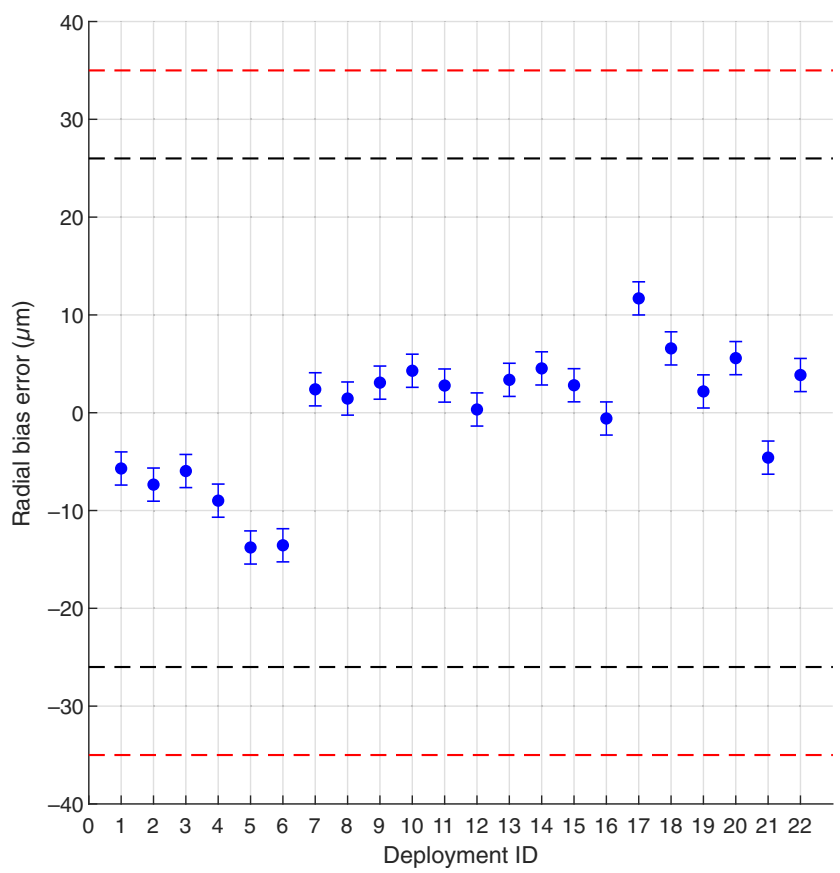

Fig. 20 Radial bias errors for each of the 22 deployments. The black dashed lines represent the $\pm 26 \mu \mathrm{m}$ tolerance interval constructed from the measured data. The red dashed lines represent the $\pm 35 \mu \mathrm{m}$ allocation.

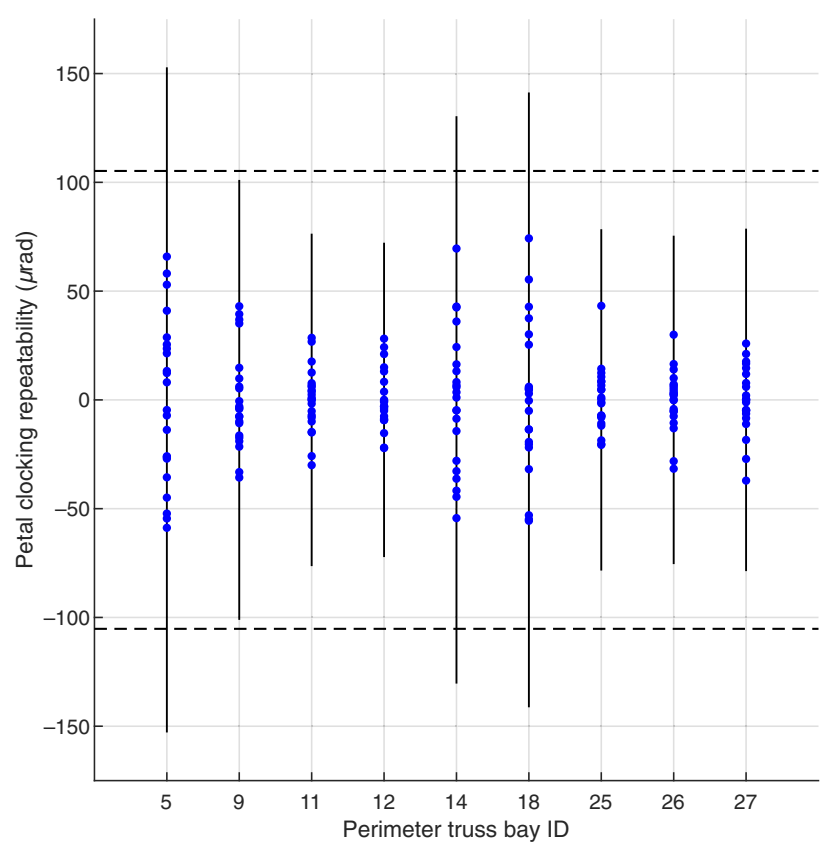

Fig. 21 Petal clocking repeatability for the 9 perimeter truss bays where clocking was measured, over the 22 deployments. The bars represent $3 \sigma$-equivalent tolerance intervals for each bay, and the black dashed lines at $\pm 105 \mu$ rad represent the overall tolerance interval for petal clocking repeatability.

accuracy errors $\overline{\Delta R}_{i}$ shown in Fig. 18), the corresponding $3 \sigma$ clocking shimming error $e_{\theta}$ is calculated as

$$
e_{\theta}=\frac{\sqrt{2} e_{R}}{L}
$$


where $L=1.25 \mathrm{~m}$ is the separation between the two petal interfaces that control clocking in a flight-like starshade design. This gives the $3 \sigma$ clocking shimming error $e_{\theta}=100 \mu \mathrm{rad}$.

Quadrature-summing the measured clocking repeatability $105 \mu \mathrm{rad}$ and the expected clocking shimming errors gives the $3 \sigma$ clocking accuracy as $145 \mu \mathrm{rad}$.

\section{Discussion of Results}

\subsection{Petal Width Results}

As shown in Fig. 16, the petal width change is very nearly constant and close to zero over the length of the petal. The uncertainty in petal width change is also constant over the length. Similarly, as shown in Fig. 17, the petal width strain is close to zero over the length of the petal, with the uncertainty in petal strain increasing at the tip due to a reduction in petal width.

Compared to the $\pm 26 \mathrm{ppm}$ requirement for petal width strain bias, the measured petal test article performance of $-0.55_{-7.30}^{+6.91} \mathrm{ppm}$ has at least $231 \%$ margin, expressed as percent allowable growth.

\subsection{Inner Disk Deployment Accuracy}

As can be seen in Figs. 18 and 20, the measured petal position accuracy errors and the associated $3 \sigma$ bounds fall within the required allocations. The petal clocking accuracy computed in Sec. 6.2 also falls within the allocation. Table 1 lists the required allocations, the corresponding measured $3 \sigma$ bounds, and the margins computed as \% allowable growth. As can be seen, all error components have at least $24 \%$ margin.

As can be seen in Fig. 18, the bounds on the radial and tangential random components are constrained primarily by 3 of the measured 34 petal interfaces. These are the only petal interfaces with the radial and tangential components of the mean accuracy error $>50 \mu \mathrm{m}$. These petal interfaces represent the worst-shimmed of the 34 petal interface measured. Through better shimming, their mean accuracy errors could be reduced. Such improved shimming is clearly possible, given the performance of the other 31 of the petal interfaces.

Based on Fig. 19, the in-plane deployment repeatability errors and the tolerance intervals are contained within $\pm 86 \mu \mathrm{m}$. This represents the deployment accuracy achievable with perfect shimming. Better shimming can be achieved by conducting more rounds of deployment and measurement to reduce uncertainty in $\overline{\mathbf{p}}_{i}$, using smaller increments of shim correction to "creep up" on the desired shim state, and using multiple or more capable laser trackers to reduce measurement uncertainty during shimming.

This result is relevant to scaling up the inner disk for larger-diameter starshades, e.g., the 52m-diameter HabEx starshade. Although deployment repeatability errors can reasonably be expected to grow with the diameter of the inner disk, shimming errors should not grow similarly.

The four components of petal position error discussed in this paper-the three random inplane rigid-body petal position errors (two translations and one rotation) and one coordinated mode of petal position errors (coordinated radial position errors) - are the most critical. There

Table 1 Required allocations for the petal position accuracy errors and the measured $3 \sigma$ bounds.

\begin{tabular}{lccc}
\hline \hline & Allocation & $\begin{array}{c}\text { Measured } \\
3 \sigma \text { bound }\end{array}$ & $\begin{array}{c}\% \\
\text { margin }\end{array}$ \\
\hline Radial bias & $35 \mu \mathrm{m}$ & $26 \mu \mathrm{m}$ & 35 \\
Radial random & $150 \mu \mathrm{m}$ & $121 \mu \mathrm{m}$ & 24 \\
Tangential random & $120 \mu \mathrm{m}$ & $91 \mu \mathrm{m}$ & 32 \\
Clocking random & $180 \mu \mathrm{rad}$ & $145 \mu \mathrm{rad}$ & 24 \\
\hline \hline
\end{tabular}


exist other modes of coordinated petal position errors, in which the petal position error components are correlated to each other. For example, if the inner disk is deformed such that the perimeter truss adopts an elliptical shape, then the radial position error of a given petal is related to its in-plane rotation error. In the experiment, such coordinated in-plane modes of petal position error were observed at low amplitudes (e.g., $33 \mu \mathrm{m}$ of ellipticity, $29 \mu \mathrm{m}$ of trefoil, $26 \mu \mathrm{m}$ of quatrefoil, and higher-order modes). The low amplitudes of these modes and also the low sensitivity of instrument contrast to these modes result in their expected contribution being less than $2.5 \%$ of the total expected contrast degradation due to inner disk deployment errors.

\subsection{Validity of Partial Inner Disk Stows}

Based on the measured data, the validity of the approach of using partial inner disk stows can be evaluated. This can be done by examining changes in the distribution of the repeatability errors as the stow percent is varied. The reason for examining repeatability errors as opposed to accuracy errors is that since the repeatability errors inherently have zero mean, all measured petal interfaces errors can be lumped into a single distribution. The accuracy errors for the petal interfaces have different means, and this collective examination would be impossible.

The in-plane repeatability errors of the petal interfaces plotted in Fig. 19 are colored according to the associated stow percent. In this plot, the four different stow percents used $(8 \%, 49 \%$, $82 \%$, and $96 \%$ ) seem to exhibit similar spreads in deployment repeatability. For a clearer comparison, Figure 22 compares the distribution of these components in a histogram format. Figure 22 shows the relative frequency of repeatability figures. Relative frequency is the raw count divided by the total number of SMR measurements for that stow percent. This allows for the comparison of the spreads in repeatability across the different stow percents, even though each stow percent has a different number of deployments associated with it (and thus a different number of SMR measurements).

Based on Fig. 22, it can be seen that there is little appreciable difference in the repeatability data between the four different stow percents used. This validates the use of partial deployments in this experiment and indicates that the final deployed position of the inner disk is set only in the final few percent of deployment.

\section{Conclusions}

The deployment performance of key starshade subsystems - the petal and the inner disk-was experimentally demonstrated using test articles at relevant scales and at relevant fidelity of design. The petal and the inner disk test articles were found to meet deployment accuracy requirements that are consistent with obtaining sufficient starlight suppression to detect and
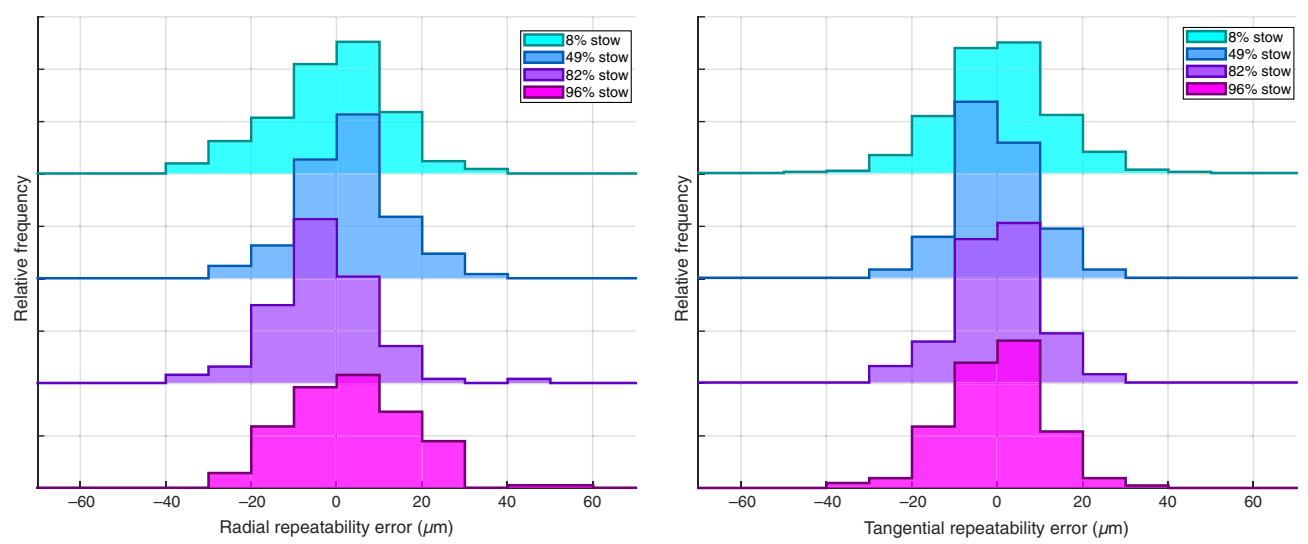

Fig. 22 Distribution of repeatability errors across all measured petal interfaces, broken down by stow fraction. Since different numbers of deployments were performed for each stow fraction, relative frequency is plotted instead of raw counts. 
study Earth-like exoplanets in the habitable zone of nearby Sun-like stars. These requirements were met with large margins.

In addition to the margins computed against the petal and inner disk deployment allocations, the instrument contrast error budget ${ }^{2}$ has large reserves at a higher level that account for differences between current designs and flight-like starshades.

This work constitutes an important demonstration of the deployment repeatability of the starshade petal and the starshade inner disk (with an integrated optical shield). The petal and inner disk test articles described here are important engineering prototypes for the starshade designs for the SRM concept and for the HabEx concept.

\subsection{Future Work}

These demonstrations were conducted as a part of the S5 activity and represent interim milestones in the path to maturing starshade mechanical technologies to TRL5. Future demonstrations are planned that will complete this maturation to TRL5. These future demonstrations will use higher-fidelity test articles: a petal test article with all features (e.g., opacity blanket and launch restraint hardware), and an inner disk test article with a higher-fidelity optical shield and with a number of petals and "petal stubs" attached to the inner disk perimeter truss. These higherfidelity test articles will be tested for deployment accuracy using methods similar to the ones presented here.

Work is also under way to simulate the deployment behavior of the petal and the inner disk using computational structural finite-element analysis (FEA) models. These FEA models will be compared against hardware performance and be used to study the starshade deployment behavior in a space environment.

\section{Appendix A: Monte Carlo Calculation of Standard Deviation of Inner Disk Deployment Accuracy}

An MC analysis was done to estimate the $3 \sigma$ levels for the radial and tangential components of the accuracy error. As defined in Fig. 15 and Eq. (13), the experimental accuracy error $\Delta_{i j}$ of the $i$ 'th petal interface at the $j$ 'th deployment is the sum of the shimming error, i.e., the mean accuracy error $\bar{\Delta}_{i}$ and the repeatability error $\mathbf{D}_{i j}$ :

$$
\Delta_{i j}=\bar{\Delta}_{i}+\mathbf{D}_{i j}
$$

This property was used to generate a population of MC accuracy errors. A population of 1000 "shim states" of the inner disk was generated. Each shim state consists of shimming errors $\bar{\Delta}_{\mathrm{MC}, i p}$ for each of the 34 measured petal interfaces, $i=1,2, \ldots, 34$ and $p=1,2, \ldots, 1000$. For each shim state, a population of 1000 deployment repeatability errors $\mathbf{D}_{\mathrm{MC}, i q}$ was generated for each petal interface, where $i=1,2, \ldots, 34$ and $q=1,2, \ldots, 1000$. This yields a population of $3.4 \times 10^{7} \mathrm{MC}$ accuracy errors $\Delta_{\mathrm{MC}, i p q}$ associated with the $p^{\prime}$ th shim state, the $q^{\prime}$ th deployment, and the $i$ 'th petal interface:

$$
\Delta_{\mathrm{MC}, i p q}=\bar{\Delta}_{\mathrm{MC}, i p}+\mathbf{D}_{\mathrm{MC}, i q} .
$$

To generate the 1000 shim states, the radial and tangential components of the shimming error for each petal interface $\bar{\Delta}_{\mathrm{MC}, i p}$ were drawn from zero-mean normal distributions:

$$
\overline{\Delta R}_{\mathrm{MC}, i p} \sim \mathcal{N}\left(0, \sigma_{\mathrm{MC}, \overline{\Delta R}}^{2}\right) \quad \overline{\Delta T}_{\mathrm{MC}, i p} \sim \mathcal{N}\left(0, \sigma_{\mathrm{MC}, \overline{\Delta T}}^{2}\right),
$$

where $\sigma_{\mathrm{MC}, \overline{\Delta R}}$ and $\sigma_{\mathrm{MC}, \overline{\Delta T}}$ are the estimated standard deviations of the radial and tangential components of the shimming errors. The standard deviations used for the MC analysis are larger than the experimentally measured standard deviations, to account for the low sample size $(n=34$ petal interfaces). The experimentally measured shimming errors are indicated by the blue squares in Fig. 18. Tolerance intervals are used to estimate the MC standard deviations. 
Table 2 Shimming error standard deviations.

\begin{tabular}{lcc}
\hline \hline & $\sigma_{\text {exp }}$ experimental $(\mu \mathrm{m})$ & $\sigma_{\mathrm{MC}} \mathrm{MC}(\mu \mathrm{m})$ \\
\hline Radial & 29.6 & 35.9 \\
Tangential & 21.3 & 25.9 \\
\hline \hline
\end{tabular}

Tolerance intervals with size $\pm k_{2} \sigma_{\exp }$ were calculated that contain that contain $99.73 \%$ population with $90 \%$ confidence, where $\sigma_{\exp }$ is the experimentally measured standard deviation. Using Eq. (11), $k_{2}=3.6358$. These tolerance intervals are comparable to $\pm 3 \sigma$ for a wellsampled distribution since they contain $99.73 \%$ of the population. By setting $k_{2} \sigma_{\exp }=$ $3 \sigma_{\mathrm{MC}} \Rightarrow \sigma_{\mathrm{MC}}=1.212 \sigma_{\mathrm{exp}}$, the standard deviations can be estimated for this MC analysis. Table 2 lists the experimental and the MC standard deviations of the shimming errors.

For each MC shim state, 1000 radial and tangential components of the repeatability errors for each petal interface were drawn from zero-mean normal distributions:

$$
\mathrm{DR}_{\mathrm{MC}, i q} \sim \mathcal{N}\left(0, \sigma_{\mathrm{MC}, \mathrm{DR} i}^{2}\right) \quad \mathrm{DT}_{\mathrm{MC}, i q} \sim \mathcal{N}\left(0, \sigma_{\mathrm{MC}, \mathrm{DT} i}^{2}\right),
$$

where $\sigma_{\mathrm{MC}, \mathrm{DR} i}$ and $\sigma_{\mathrm{MC}, \mathrm{DT} i}$ are the estimated standard deviations of the radial and tangential components of the repeatability errors. These estimated standard deviations are different for each petal interface and are calculated, as above, using tolerance intervals:

$$
3 \sigma_{\mathrm{MC}, \mathrm{DR} i}=k \hat{\sigma}_{R i} \quad 3 \sigma_{\mathrm{MC}, \mathrm{DT} i}=k \hat{\sigma}_{T i},
$$

where $\hat{\sigma}_{R i}$ and $\hat{\sigma}_{T i}$ are the corrected sample standard deviations and $k=3.8596$ is the tolerance interval factor. These quantities are defined in Sec. 5.2.1. The estimated standard deviations of the repeatability errors used in this MC analysis ranged between 9.2 and $28.6 \mu \mathrm{m}$ for the radial component and between 8.4 and $25.5 \mu \mathrm{m}$ for the tangential component for the 34 petal interfaces.

This process yields 1000 shim states for the 34 petal interfaces. Each shim state is then "deployed" 1000 times, resulting in a population of $3.4 \times 10^{7}$ radial and tangential components of the accuracy error. The standard deviations of these populations gives the $3 \sigma$ bounds for the

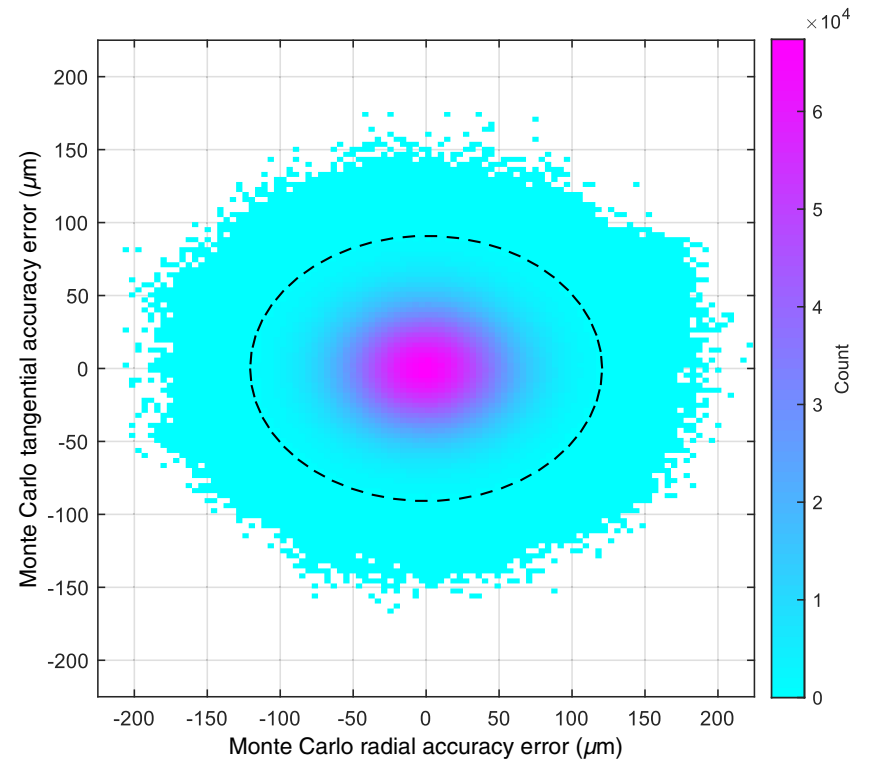

Fig. 23 Histogram showing the results of the $M C$ analysis. The major and minor axes of black dashed ellipse represents the computed $3 \sigma$ radial and tangential accuracy errors $-121 \mu \mathrm{m}$ radial and $91 \mu \mathrm{m}$ tangential. 
measured accuracy errors: $121 \mu \mathrm{m}$ for the radial component and $91 \mu \mathrm{m}$ for the tangential component. Figure 23 shows the results of this MC analysis.

\section{Acknowledgments}

The research was carried out at the Jet Propulsion Laboratory, California Institute of Technology, under a contract with the National Aeronautics and Space Administration (No. 80NM0018D0004). The authors declare no relevant financial interests in the manuscript and no potential conflicts of interest.

\section{References}

1. W. Cash, "Detection of Earth-like planets around nearby stars using a petal-shaped occulter," Nature 442(7098), 51-53 (2006).

2. P. Willems, "Starshade to TRL5 (S5) technology development plan," Tech. Rep., Jet Propulsion Laboratory, California Institute of Technology (2018).

3. M. Arya et al., "Demonstration of deployment accuracy of the starshade inner disk subsystem," in AIAA Scitech 2020 Forum, Orlando, FL (2020).

4. M. Arya et al., "Starshade technology development activity milestone 7C: demonstration of deployment accuracy of the starshade inner disk subsystem," Tech. Rep. (2020).

5. F. Mechentel et al., "Starshade technology development activity milestone 5A: verify petal pre-launch accuracy," Tech. Rep., Jet Propulsion Laboratory, California Institute of Technology (2020).

6. S. Seager et al., "Starshade Rendezvous Probe," Tech. Rep. (2019).

7. B. S. Gaudi et al., "The Habitable Exoplanet Observatory (HabEx)," Proc. SPIE 11115, 111150M (2019).

8. M. W. Thomson et al., "Starshade design for occulter based exoplanet missions," Proc. SPIE 7731, 773153 (2010).

9. D. Webb et al., "Starshade mechanical architecture and technology effort," in 3rd AIAA Spacecraft Struct. Conf., San Diego, California (2016).

10. D. Webb et al., "Advances in starshade technology readiness for an exoplanet characterizing science mission in the 2020's," Proc. SPIE 9912, 99126H (2016).

11. M. Arya et al., "Starshade mechanical design for the Habitable Exoplanet imaging mission concept (HabEx)," Proc. SPIE 10400, 104001C (2017).

12. D. Webb et al., "Advances in starshade technology readiness for an exoplanet characterizing science mission in the 2020's," Proc. SPIE 11117, 111170P (2019).

13. A. G. Roederer, "Historical overview of the development of space antennas," Chapter 7 in Space Antenna Handbook, W. A. Imbriale, S. S. Gao, and L. Boccia, Eds., pp. 271-272, John Wiley and Sons, Ltd., West Sussex (2012).

14. T. G. Campbell et al., "Development of the 15-meter hoop/column antenna system," Large Space Systems Technology, pp. 167-212 (1984).

15. M. Thomson, "The AstroMesh deployable reflector," IEEE Antennas and Propag. Soc. Int. Symp. Dig., Vol. 3, pp. 1516-1519 (1999).

16. N. J. Kasdin et al., "Advancing technology for starlight suppression via an external occulter," TDEM Report (2012).

17. N. J. Kasdin et al., "Verifying deployment tolerances of an external occulter for starlight suppression," TDEM Report (2014).

18. D. Webb et al., "Successful Starshade petal deployment tolerance verification in support of NASA's technology development for exoplanet missions," Proc. SPIE 9151, 91511P (2014).

19. B. Hirsch et al., "Starshade deployable inner disk subsystem structure design and development," in 3rd AIAA Spacecraft Struct. Conf., American Institute of Aeronautics and Astronautics, San Diego, California (2016).

20. S. B. Shaklan et al., "Error budgets for the Exoplanet Starshade (Exo-S) probe-class mission study," Proc. SPIE 9605, $96050 \mathrm{Z}$ (2015). 
21. J. Steeves et al., "Precision optical edges for a starshade external occulter," Proc. SPIE 9912, 991220 (2016).

22. J. Steeves et al., "Development of low-scatter optical edges for starshades," Proc. SPIE 10706, 107065K (2018).

23. E. Hilgemann et al., "Advancements in precision edges for a starshade external occulter," Proc. SPIE 11117, 111170Q (2019).

24. L. D. Peterson and M. S. Lake, "Micron accuracy deployment experiment (MADE)," Tech. Rep. NASA-CR-197792 (1995).

25. B. Kozola et al., "Experimental characterization of deployment repeatability for opticalprecision space structures," in 19th AIAA Appl. Aerodynamics Conf., American Institute of Aeronautics and Astronautics, Anaheim, CA (2001).

26. O. Stohlman, M. Silver, and D. Waller, "Deployment repeatability," Chapter 5 in Testing Large Ultra-Lightweight Spacecraft, J. A. Banik and C. H. Jenkins, Eds., pp. 133-172, American Institute of Aeronautics and Astronautics, Reston, VA (2017).

27. D. Webb et al., "Starshade technology development activity milestone 8A: verify petal position on-orbit stability," Tech. Rep., Jet Propulsion Laboratory, California Institute of Technology (2020).

28. S. A. Zirbel et al., "Accommodating thickness in origami-based deployable arrays," J. Mech. Des. 135, 111005 (2013).

29. D. Sigel et al., "Application of origami in starshade spacecraft blanket design," in Proc. ASME 38th Mech. and Rob. Conf., Buffalo, New York (2014).

30. Micro-Vu Corporation, "EXCEL measuring center" (2007).

31. A. Hockman and D. W. Kessler, "Thermal and moisture expansion studies of some domestic granites," J. Res. Nat. Bur. Stand. 44, 395-410 (1950).

32. R. A. Paquin, "Materials for optical systems," in Optomechanical Engineering Handbook, A. Ahmad, Ed., CRC Press LLC, Boca Raton, Florida (1999).

33. Leica Geosystems, "Leica AT402 user manual" (2013).

34. Leica Geosystems, "Leica absolute tracker AT401 white paper" (2010).

35. E. Cady, "Boundary diffraction wave integrals for diffraction modeling of external occulters," Opt. Express 20(14), 15196-15208 (2012).

36. J. Ding, Private Communication (2020).

37. W. A. Jensen, "Approximations of tolerance intervals for normally distributed data," Qual. Reliab. Eng. Int. 25, 571-580 (2009).

Biographies of the authors are not available. 